\title{
Internal dynamics of the massive cluster Abell 697: a multiwavelength analysis
}

\author{
M. Girardi ${ }^{1,2}$, W. Boschin ${ }^{1,3}$, and R. Barrena ${ }^{4}$ \\ 1 Dipartimento di Astronomia, Università degli Studi di Trieste, via Tiepolo 11, 34131 Trieste, Italy \\ e-mail: girardi@oats.inaf.it \\ 2 INAF - Osservatorio Astronomico di Trieste, via Tiepolo 11, 34131 Trieste, Italy \\ 3 Fundación Galileo Galilei - INAF, C/Alvarez de Abreu 70, 38700 Santa Cruz de La Palma, Canary Islands, Spain \\ ${ }^{4}$ Instituto de Astrofisica de Canarias, C/via Lactea s/n, 38200 La Laguna, Tenerife, Canary Islands, Spain
}

Received 14 February 2006 / Accepted 19 April 2006

ABSTRACT

\begin{abstract}
Aims. We conduct an intensive study of the rich, X-ray luminous, and hot galaxy cluster Abell 697 (at $z=0.282$ ), likely containing a diffuse radio-emission, to determine its dynamical status.

Methods. Our analysis is based on new spectroscopic data obtained at the TNG telescope for 93 galaxies and on new photometric data obtained at the INT telescope in a large field. We combine galaxy velocity and position information to select 68 cluster members (out to $\sim 1.3 h_{70}^{-1} \mathrm{Mpc}$ from the cD galaxy), determine global dynamical properties, and detect possible substructures. The investigation of the dynamical status is also performed by using X-ray data stored in the Chandra archive.

Results. We compute the line-of-sight (LOS) velocity dispersion of galaxies, $\sigma_{v}=1334_{-95}^{+114} \mathrm{~km} \mathrm{~s}^{-1}$, in agreement with the high average X-ray temperature $T_{\mathrm{X}}=(10.2 \pm 0.8) \mathrm{keV}$ recovered from Chandra data, as expected in the case of energy-density equipartition between galaxies and gas. Assuming that the cluster is in dynamical equilibrium and mass follows the galaxy distribution, we find that $\mathrm{A} 697$ is a very massive cluster obtaining $M\left(<R_{\max }=0.75 h_{70}^{-1} \mathrm{Mpc}\right)=9.5_{-1.5}^{+1.8} \times 10^{14} h_{70}^{-1} M_{\odot}$ and $M\left(<R_{\mathrm{vir}}=3.85 h_{70}^{-1} \mathrm{Mpc}\right)=$ $4.5_{-1.3}^{+1.4} \times 10^{15} h_{70}^{-1} M_{\odot}$ for the region well sampled by the spectroscopic data and for the entire virialized region, respectively. Further investigations find that A697 is not fully relaxed, as shown by the non Gaussianity of the velocity distribution, the elongation of the $\mathrm{X}$-ray emission, and the presence of small-size substructures in the central region.

Conclusions. Our results suggest that we are observing a cluster that has undergone a complex cluster merger occurring mainly along the LOS, with a transverse component in the SSE-NNW direction. The importance and the phase of the merging event is discussed. The spatial correlation between the (likely) radio halo and the optical and X-ray cluster structure supports the hypothesis of a relation between extended radio emission and merging phenomena.
\end{abstract}

Key words. galaxies: clusters: general - galaxies: clusters: individual: Abell 697 - galaxies: distances and redshifts galaxies: intergalactic medium - cosmology: observations

\section{Introduction}

Clusters of galaxies are recognized to be not simple relaxed structures, but rather as evolving via merging processes in a hierarchical fashion from poor groups to rich clusters. Much progress has been made in recent years in the observations of the signatures of merging processes (see Feretti et al. 2002, for a general review). The presence of substructure, which is indicative of a cluster in an early phase of the process of dynamical relaxation or of secondary infall of clumps into already virialized clusters, occurs in about $50 \%$ of clusters as shown by optical and X-ray data (e.g., Geller \& Beers 1982; Mohr et al. 1996; Girardi et al. 1997; Kriessler \& Beers 1997; Jones \& Forman 1999; Schuecker et al. 2001; Burgett et al. 2004).

A new aspect of these investigations is the possible connection of cluster mergers with the presence of extended, diffuse radio sources, halos and relics. They are rare, large (up to $\sim 1 h_{70}^{-1} \mathrm{Mpc}$ ), amorphous cluster sources of uncertain origin and generally steep radio spectra (Hanisch 1982; see also Giovannini \& Feretti 2002, for a recent review). They appear to be associated with very rich clusters that have undergone recent mergers and thus it has been suggested by various authors that cluster halos/relics are related to recent merger activity (e.g., Tribble 1993; Burns et al. 1994; Feretti 1999).

The synchrotron radio emission of halos and relics demonstrates the existence of large scale cluster magnetic fields, of the order of $0.1-1 \mu \mathrm{G}$, and of widespread relativistic particles of energy density $10^{-14}-10^{-13} \mathrm{erg} \mathrm{cm}^{-3}$. The difficulty in explaining radio-halos arises from the combination of their large size and the short synchrotron lifetime of relativistic electrons. The expected diffusion velocity of the electron population is on the order of the Alfven speed $\left(\sim 100 \mathrm{~km} \mathrm{~s}^{-1}\right)$ making it difficult for the electrons to diffuse over a megaparsec-scale region within their radiative lifetime. Therefore, one needs a mechanism by which the relativistic electron population can be transported over large distances in a short time, or a mechanism by which the local electron population is reaccelerated and the local magnetic fields are amplified over an extended region. The cluster-cluster merger can potentially supply both mechanisms (e.g., Giovannini et al. 1993; Burns et al. 1994; Röttgering et al. 1994; see also Feretti et al. 2002; Sarazin 2002, for reviews). However, the question is still debated since the diffuse radio sources are quite uncommon and only recently have we been able to study these phenomena on the basis of sufficient 
statistics ( $\sim 30$ clusters up to $z \sim 0.3$, e.g., Giovannini et al. 1999; see also Giovannini \& Feretti 2002; Feretti 2005).

Growing evidence of the connection between diffuse radio emission and cluster merging is based on X-ray data (e.g., Böhringer \& Schuecker 2002; Buote 2002). Studies based on a large number of clusters have found a significant relation between the radio and the X-ray surface brightness (Govoni et al. 2001a,b) and between the presence of radio-halos/relics and irregular and bimodal X-ray surface brightness distribution (Schuecker et al. 2001). New unprecedent insights into merging processes in radio clusters are offered by Chandra and XMM-Newton observations (e.g., Markevitch \& Vikhlinin 2001; Markevitch et al. 2002; Fujita et al. 2004; Henry et al. 2004; Kempner \& David 2004).

Optical data are a powerful way to investigate the presence and the dynamics of cluster mergers, too (e.g., Girardi \& Biviano 2002). The spatial and kinematical analysis of member galaxies allows us to detect and measure the amount of substructure, to identify and analyze possible pre-merging clumps or merger remnants. This optical information is complementary to $\mathrm{X}$-ray information since galaxies and ICM react on different time scales during a merger (see numerical simulations by Roettiger et al. 1997). Unfortunately, to date optical data are lacking or are poorly exploited. The sparse literature contains a few individual clusters (e.g., Colless \& Dunn 1996; Gómez et al. 2000; Barrena et al. 2002; Mercurio et al. 2003; Boschin et al. 2004; Boschin et al. 2006). We have conducted an intensive study of Abell 697 (hereafter A697) that has a probable diffuse radio emission located in the cluster center (Kempner \& Sarazin 2001).

A697 is one of the higher redshift clusters in the ACO cata$\log (z \sim 0.282$, Abell et al. 1989). It is a fairly rich, X-ray luminous and hot cluster: Abell richness class $=1$ (Abell et al. 1989), $L_{\mathrm{X}}(0.1-2.4 \mathrm{keV})=16.30 \times 10^{44} h_{50}^{-2} \mathrm{erg} \mathrm{s}^{-1}$ (Ebeling et al. 1998), $T_{\mathrm{X}} \sim 8-11 \mathrm{keV}$ (e.g., Metzger \& Ma 2000, hereafter M00; White 2000; Ota \& Mitsuda 2004; Bonamente et al. 2005). It shows an arclike feature as revealed by Keck images (M00) and is one of the most massive clusters analyzed by Dahle et al. (2002) through the weak lensing analysis. Observational signatures of the young dynamical state of A697 come from Keck images of the dominant galaxy, a cD showing a highly asymmetric halo and a (likely) secondary nucleus (M00). Both X-ray emission and gravitational lensing data indicate an elongated mass distribution (M00; Dahle et al. 2002; De Filippis et al. 2005) as expected in a merger collision (e.g., Roettiger et al. 1996).

To date few spectroscopic data have been reported in the literature. Crawford et al. (1995) measured the redshift for the cD galaxy. M00 measured redshift for another 7(9) member galaxies giving an uncertain value of 553(941) $\mathrm{km} \mathrm{s}^{-1}$ for the line-of-sight (hereafter LOS) velocity dispersion. Recently, we have carried out spectroscopic observations with the TNG telescope giving new redshift data for 93 galaxies in the field of A697, as well as photometric observations at the INT telescope. Our present analysis is based on these optical data and $\mathrm{X}$-ray Chandra archival data.

This paper is organized as follows. We present the new optical data in Sect. 2 and the relevant analyses in Sects. 3 and 4. Our analysis of X-ray Chandra data is shown in Sect. 5. We discuss the dynamical state of A697 in Sect. 6 and summarize our results in Sect. 7.

Unless otherwise stated, we give errors at the $68 \%$ confidence level (hereafter c.l.). Throughout the paper, we assume a flat cosmology with $\Omega_{\mathrm{m}}=0.3, \Omega_{\Lambda}=0.7$ and $H_{0}=$ $70 h_{70} \mathrm{~km} \mathrm{~s}^{-1} \mathrm{Mpc}^{-1}$. For this cosmological model, $1^{\prime}$ corresponds to $256 h_{70}^{-1} \mathrm{kpc}$ at the cluster redshift.

\section{Data sample}

Multi-object spectroscopic observations of A697 were carried out at the TNG telescope in December 2003 during the program of proposal AOT8/CAT-G6. We used DOLORES/MOS with the LR-B Grism 1, yielding a dispersion of $187 \AA / \mathrm{mm}$, and the Loral CCD of $2048 \times 2048$ pixels (pixel size of $15 \mu \mathrm{m}$ ). This combination of grating and detector results in dispersions of $2.8 \AA /$ pix. We have taken three MOS masks for a total of 114 slits. We acquired two exposures of $1800 \mathrm{~s}$ for two masks and three exposures of $1800 \mathrm{~s}$ for the last one. Wavelength calibration was performed using Helium-Argon lamps.

Reduction of spectroscopic data was carried out with IRAF $^{1}$ package.

Radial velocities were determined using the crosscorrelation technique (Tonry \& Davis 1979) implemented in the RVSAO package (developed at the Smithsonian Astrophysical Observatory Telescope Data Center). Each spectrum was correlated against six templates for a variety of galaxy spectral types: E, S0, Sa, Sb, Sc, Ir (Kennicutt 1992). The template producing the highest value of $\mathcal{R}$, i.e., the parameter given by RVSAO and related to the signal-to-noise of the correlation peak, was chosen. Moreover, all spectra and their best correlation functions were examined visually to verify the redshift determination. In some cases (IDs 1, 30, 75, 81; see Table 1) we took the EMSAO redshift, i.e. that determined from the emission lines in the spectra, as a reliable estimate of the redshift. One object $\left(\mathrm{RA}=08^{\mathrm{h}} 43^{\mathrm{m}} 08^{\mathrm{s}} \cdot 16\right.$, Dec $=+36^{\circ} 24^{\prime} 39^{\prime}$. $1(\mathrm{~J} 2000.0)$, see the diamond in Fig. 1) was found to be a quasar at $z \sim 1.50$ (see also the point-like X-ray emission in the upper-left corner of Fig. 2) and has not been considered in our analysis.

For three galaxies we obtained two redshift determinations, which are of similar quality. We compared the two determinations computing the mean and the rms of the variable $z_{1}-$ $\left.z_{2}\right) / \sqrt{\operatorname{err}_{1}^{2}+\operatorname{err}_{2}^{2}}$, where $z_{1}$ comes from MOS 1 and $z_{2}$ from MOS 2 (or MOS 3). We obtained a mean $=0.23 \pm 0.43$ and a rms $=0.74$, to be compared with the expected values of 0 and 1 . The resulting mean shows that two sets of measurements are consistent with having the same velocity zero-point, and the value of rms is compatible with a value of 1 according to the $\chi^{2}$-test. For the three galaxies we used the average of the two redshift determinations and the corresponding error.

Our spectroscopic catalog consists of 93 galaxies out to a radius of $R \sim 5^{\prime}$ from the cD galaxy (ID 41 ).

Our photometry observations were carried out with the Wide Field Camera (WFC), mounted at the prime focus of the $2.5 \mathrm{~m}$ INT telescope (located at Roque de los Muchachos Observatory, La Palma, Spain). We observed A697 on December 18th 2004 under photometric conditions with a seeing of about $2^{\prime \prime}$.

The WFC consists of a 4 CCD mosaic covering a $30^{\prime} \times$ $30^{\prime}$ field of view, with only a $20 \%$ marginally vignetted area. We took 10 exposures of $720 \mathrm{~s}$ in $B_{\mathrm{H}}$ and $360 \mathrm{~s}$ in $R_{\mathrm{H}}$ Harris filters (a total of $7200 \mathrm{~s}$ and $3600 \mathrm{~s}$ in each band) developing a dithering pattern of ten positions. This observing mode allowed us to build a "supersky" frame that was used to correct our images for fringing patterns (Gullixson 1992). In addition, the dithering helped us to clean cosmic rays and avoid gaps between the CCDs in the final images. The complete reduction process (including flat fielding, bias subtraction and bad-columns elimination) yielded

1 IRAF is distributed by the National Optical Astronomy Observatories, which are operated by the Association of Universities for Research in Astronomy, Inc., under cooperative agreement with the National Science Foundation. 
Table 1. Velocity catalog of 93 spectroscopically measured galaxies. In Col. 1, IDs in italics indicate non-cluster galaxies.

\begin{tabular}{|c|c|c|c|c|c|c|}
\hline ID & $\begin{array}{c}\alpha, \delta(\mathrm{J} 2000) \\
08^{\mathrm{h}},+36^{\circ}\end{array}$ & $B$ & $R$ & $\begin{array}{r}v \\
\mathrm{~km}\end{array}$ & $\begin{array}{c}\Delta v \\
\mathrm{~s}^{-1}\end{array}$ & EL \\
\hline 1 & $4235.42,2058.0$ & 21.77 & 20.76 & 109714 & 26 & [OII] \\
\hline 2 & $4236.31,2143.0$ & 21.33 & 19.21 & 84766 & 91 & \\
\hline 3 & $4237.68,2149.0$ & 21.05 & 18.77 & 84801 & 46 & \\
\hline 4 & $4239.89,2151.4$ & 21.45 & 19.50 & 85868 & 54 & \\
\hline 5 & $4242.00,2213.7$ & 22.26 & 19.94 & 84593 & 53 & \\
\hline 6 & $4242.38,2256.9$ & 22.51 & 20.25 & 87591 & 68 & \\
\hline 7 & $4243.13,2245.4$ & 23.07 & 20.41 & 85957 & 82 & \\
\hline 8 & $4245.96,2328.2$ & 19.37 & 18.49 & 9076 & 63 & {$[\mathrm{OII}],[\mathrm{OIII}]$} \\
\hline 9 & $4246.15,2022.2$ & 22.96 & 20.55 & 85453 & 95 & \\
\hline 10 & $4246.15,2456.2$ & 22.50 & 20.05 & 154872 & 43 & \\
\hline 11 & $4246.32,2445.8$ & 22.22 & 19.95 & 83814 & 48 & \\
\hline 12 & $4247.83,2411.7$ & 21.77 & 19.37 & 85195 & 52 & \\
\hline 13 & 2221.8 & 20.90 & 19.70 & 82551 & 250 & [OII] \\
\hline 14 & $4248.34,1840.8$ & 20.64 & 19.69 & 15597 & 127 & [OIII], $\mathrm{H} \alpha$ \\
\hline 15 & $4248.38,2236.4$ & 21.57 & 19.67 & 81170 & 82 & \\
\hline 16 & $4248.67,1714.4$ & 21.40 & 19.33 & 85836 & 65 & \\
\hline 17 & $4249.46,1652.1$ & 21.45 & 18.80 & 103561 & 89 & \\
\hline 18 & $4249.97,2235.7$ & 21.97 & 19.59 & 86779 & 58 & \\
\hline 19 & $4250.16,2440.6$ & 21.43 & 19.07 & 87839 & 60 & \\
\hline 20 & $4250.36,2312.1$ & 21.80 & 19.49 & 82694 & 75 & \\
\hline 21 & $4251.31,2317.8$ & 20.88 & 18.85 & 102192 & 56 & \\
\hline 22 & $4251.36,1832.2$ & 22.21 & 19.70 & 84197 & 82 & \\
\hline 23 & $4251.38,2023.9$ & 20.06 & 18.25 & 41336 & 41 & \\
\hline 24 & $4251.91,1837.9$ & 22.59 & 20.09 & 83253 & 63 & \\
\hline 25 & $4252.87,2231.8$ & 22.48 & 20.06 & 83404 & 68 & \\
\hline 26 & $4252.99,1951.0$ & 20.37 & 18.81 & 86695 & 49 & \\
\hline 27 & $4253.33,2147.8$ & 22.23 & 19.59 & 85917 & 75 & \\
\hline 28 & $4253.47,2338.5$ & 22.16 & 19.73 & 82697 & 77 & \\
\hline 29 & $4253.69,2136.3$ & 21.76 & 19.42 & 87319 & 74 & \\
\hline 30 & $4253.93,1916.6$ & 22.24 & 21.05 & 101857 & 311 & {$[\mathrm{OII}],[\mathrm{OIII}]$} \\
\hline 31 & $4254.05,2416.5$ & 21.42 & 18.86 & 85853 & 43 & \\
\hline 32 & $4254.26,1921.2$ & 22.46 & 20.30 & 101888 & 57 & \\
\hline 33 & $4254.36,2103.5$ & 21.37 & 19.83 & 82240 & 53 & \\
\hline 34 & $4254.67,2114.2$ & 21.94 & 19.99 & 85332 & 43 & \\
\hline 35 & $4255.39,2122.1$ & 22.30 & 19.50 & 82513 & 63 & \\
\hline 36 & $4256.18,2126.7$ & 21.90 & 19.04 & 85475 & 41 & \\
\hline 37 & $4256.98,2153.3$ & 22.00 & 19.82 & 85011 & 97 & \\
\hline 38 & $4257.12,2422.6$ & 20.47 & 18.47 & 7199 & 33 & \\
\hline 39 & 4257.3 & 21.50 & 19.22 & 82900 & 66 & \\
\hline 40 & $4257.38,2057.4$ & 21.50 & 19.43 & 87821 & 49 & \\
\hline 41 & $4257.55,2159.9$ & 20.48 & 17.95 & 84361 & 58 & \\
\hline 42 & $4257.60,2201.2$ & 21.90 & 19.74 & 84711 & 45 & \\
\hline 43 & $4257.62,2136.5$ & 22.18 & 19.49 & 82422 & 67 & \\
\hline 44 & $4257.84,2429.0$ & 21.16 & 18.77 & 84988 & 43 & \\
\hline 45 & $4258.01,2245.3$ & 20.95 & 18.30 & 83468 & 48 & \\
\hline 46 & $4258.13,2127.3$ & 21.66 & 19.41 & 85455 & 85 & \\
\hline 47 & $4258.13,2219.5$ & 21.06 & 18.65 & 84221 & 37 & \\
\hline 48 & $4258.44,2259.1$ & 22.35 & 19.58 & 87125 & 57 & \\
\hline 49 & $4258.56,2348.2$ & 21.59 & 19.18 & 81888 & 77 & \\
\hline 50 & $4259.21,2323.8$ & 21.83 & 19.53 & 82610 & 67 & \\
\hline 51 & $4259.38,2216.5$ & 22.20 & 19.47 & 86794 & 56 & \\
\hline 52 & $4259.66,2233.0$ & 22.30 & 19.55 & 84290 & 130 & \\
\hline 53 & $4259.98,2000.9$ & 21.85 & 19.44 & 86525 & 46 & \\
\hline 54 & $4300.19,1906.5$ & 22.68 & 20.34 & 82625 & 73 & \\
\hline 55 & $4300.24,2244.9$ & 22.63 & 20.22 & 83829 & 98 & \\
\hline 56 & $4300.29,2128.4$ & 21.98 & 19.62 & 86920 & 52 & \\
\hline
\end{tabular}

a final coadded image where the variation of the sky was lower than $0.4 \%$ in the whole frame. Another effect associated with the wide field frames is the distortion of the field. In order to match the photometry of several filters, a good astrometric solution is needed to take into account these distortions. Using
Table 1. continued.

\begin{tabular}{|c|c|c|c|c|c|c|}
\hline ID & $\begin{array}{c}\alpha, \delta(\mathrm{J} 2000) \\
08^{\mathrm{h}},+36^{\mathrm{o}}\end{array}$ & $B$ & $R$ & $\begin{array}{r}v \\
\mathrm{~km}\end{array}$ & $\begin{array}{c}\Delta v \\
\mathrm{~s}^{-1}\end{array}$ & EL \\
\hline 57 & $4300.43,1745.1$ & 20.64 & 18.20 & 84509 & 48 & \\
\hline 58 & $4300.58,2200.2$ & 22.39 & 19.73 & 86018 & 71 & \\
\hline 59 & $4300.91,2532.3$ & 21.11 & 19.30 & 89555 & 75 & \\
\hline 60 & $4301.34,1824.9$ & 22.12 & 19.66 & 84617 & 61 & \\
\hline 61 & $4301.49,2433.2$ & 22.79 & 20.37 & 82420 & 55 & \\
\hline 62 & $4301.51,1942.7$ & 20.74 & 18.49 & 80752 & 39 & \\
\hline 63 & $4301.58,2356.3$ & 21.56 & 19.10 & 82712 & 51 & \\
\hline 64 & $4301.61,2403.7$ & 21.68 & 19.44 & 83882 & 59 & \\
\hline 65 & 43 01.73, 2046.1 & 21.06 & 18.79 & 85345 & 36 & \\
\hline 66 & $4302.09,1826.7$ & 22.09 & 19.83 & 84853 & 79 & \\
\hline 67 & $4302.14,2159.1$ & 19.95 & 18.05 & 79922 & 37 & [OII], [OIII] \\
\hline 68 & $4302.28,2026.4$ & 21.29 & 18.92 & 83675 & 63 & \\
\hline 69 & $4302.62,2151.0$ & 21.93 & 19.42 & 82818 & 69 & \\
\hline 70 & 43 02.66, 2137.4 & 21.78 & 19.50 & 85424 & 27 & \\
\hline 71 & 43 03.46, 2022.6 & 22.43 & 20.13 & 110946 & 98 & \\
\hline 72 & 43 03.48, 1812.6 & 22.29 & 19.72 & 84978 & 49 & \\
\hline 73 & $4303.55,2214.0$ & 21.22 & 19.75 & 85604 & 57 & \\
\hline 74 & 43 03.74, 2040.6 & 22.48 & 20.29 & 80695 & 83 & \\
\hline 75 & $4304.94,2103.3$ & 20.97 & 19.78 & 15299 & 85 & $\begin{array}{l}{[\mathrm{OII}],[\mathrm{OIII}]} \\
{[\mathrm{OI}], \mathrm{H} \alpha}\end{array}$ \\
\hline 76 & 43 05.23, 2051.6 & 21.78 & 19.63 & 82393 & 63 & \\
\hline 77 & 43 05.30, 1936.4 & 22.77 & 20.07 & 83863 & 57 & \\
\hline 78 & 43 05.47, 1914.4 & 21.48 & 18.96 & 83856 & 58 & \\
\hline 79 & $4305.50,2223.9$ & 19.89 & 18.70 & 80047 & 35 & {$[\mathrm{OII}],[\mathrm{OIII}]$} \\
\hline 80 & $4305.57,1851.9$ & 22.87 & 20.57 & 90606 & 106 & \\
\hline 81 & 43 06.22, 2519.1 & 22.25 & 20.66 & 77636 & 71 & [OII] \\
\hline 82 & $4306.45,2155.0$ & 21.23 & 18.88 & 82376 & 44 & \\
\hline 83 & $4306.46,2156.8$ & 22.72 & 20.32 & 84841 & 67 & \\
\hline 84 & 43 07.80, 2058.1 & 21.41 & & 85932 & 29 & \\
\hline 85 & 43 08.62, 2139.8 & 21.56 & 19.43 & 81414 & 54 & \\
\hline 86 & 43 09.17, 2257.2 & 22.24 & 19.67 & 87994 & 100 & \\
\hline 87 & $4311.23,1923.3$ & 20.60 & 18.64 & 87555 & 52 & \\
\hline 88 & $4311.38,2124.0$ & 22.54 & 20.11 & 85718 & 134 & \\
\hline 89 & $4312.67,2212.9$ & 21.84 & 19.45 & 83517 & 54 & \\
\hline 90 & $4315.34,2020.2$ & 18.87 & 17.71 & 32968 & 39 & $\begin{array}{l}{[\mathrm{OII}], \mathrm{H} \beta,} \\
{[\mathrm{OIII}], \mathrm{H} \alpha,[\mathrm{SII}]}\end{array}$ \\
\hline 91 & $4315.79,2345.5$ & 21.51 & 19.84 & 104134 & 83 & \\
\hline 92 & $4316.30,2014.9$ & 18.92 & 17.42 & 25661 & 41 & $\mathrm{H} \alpha,[\mathrm{SII}]$ \\
\hline 93 & $4317.04,2058.4$ & 21.46 & 20.12 & 85899 & 86 & \\
\hline
\end{tabular}

IRAF tasks and taking as a reference the USNO B1.0 catalog we were able to find an accurate astrometric solution (rms $\left.0.5^{\prime \prime}\right)$ across the full frame. The photometric calibration was performed using Landolt standard fields obtained during the observation.

We finally identified galaxies in our $B_{\mathrm{H}}$ and $R_{\mathrm{H}}$ images and measured their magnitudes with the SExtractor package (Bertin \& Arnouts 1996) and AUTOMAG procedure. In a few cases, (e.g., close companion galaxies, galaxies close to defects of CCD), the standard SExtractor photometric procedure failed. In these cases we computed magnitudes by hand. This method consists of assuming a galaxy profile of a typical elliptical and scaling it to the maximum observed value. The integration of this profile gives us an estimate of the magnitude. This method is similar to PSF photometry, but assumes a galaxy profile, more appropriate in this case.

We transformed all magnitudes into the Johnson-Cousins system (Johnson \& Morgan 1953; Cousins 1976). We used $B=B_{\mathrm{H}}+0.13$ and $R=R_{\mathrm{H}}$, as derived from the Harris filter characterization (http://www.ast.cam.ac.uk/ $\sim$ wfcsur/ technical/photom/colours/) and assuming a $B-V \sim 1.0$ 


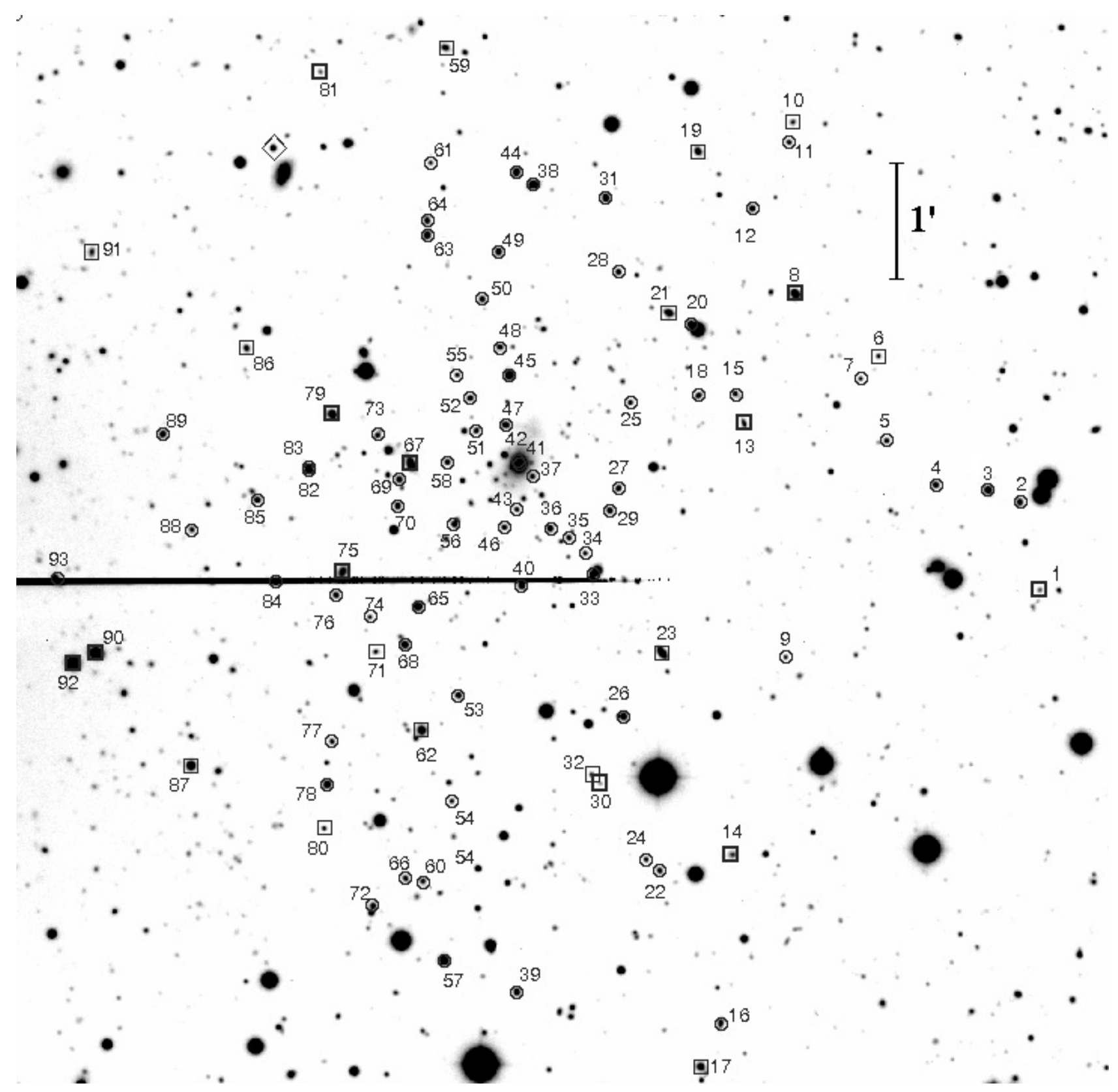

Fig. 1. $R$-band image of A697 (North at the top and East to the left) taken with the WFC camera of the INT. Galaxies with successful velocity measurements are labeled as in Table 1. Circles and boxes indicate cluster members and non-member galaxies, respectively. Out of non-member galaxies, bold-face boxes indicate emission-line galaxies. Diamond indicates a QSO at $z \sim 1.50$. The horizontal spike is due to the presence of a bright star $\sim 7^{\prime}$ East of the cluster.

for E-type galaxies (Poggianti 1997). As a final step, we estimated and corrected the galactic extinction $A_{B} \sim 0.15$, $A_{R} \sim 0.09$ from Burstein \& Heiles (1982) reddening maps.

We estimated that our photometric sample is complete down to $R=22.0$ (23.2) and $B=23.0$ (24.2) for $S / N=5$ (3) within the observed field.

We assigned $R(B)$ magnitudes to 92 (93) out of the 93 galaxies of our spectroscopic catalog. We measured redshifts for galaxies down to magnitude $R \sim 21$, but a high level of completeness is reached only for galaxies with magnitude $R<20$ ( $\sim 70 \%$ completeness).

Table 1 lists the velocity catalog (see also Fig. 1): identification number of each galaxy, ID (Col. 1); right ascension and declination, $\alpha$ and $\delta$ (J2000, Col. 2); B magnitudes (Col. 3); $R$ magnitudes (Col. 4); heliocentric radial velocities, $v=c z_{\odot}$
(Col. 5) with errors, $\Delta v$ (Col. 6); emission lines detected in the spectra (Col. 7).

\section{Analysis of the spectroscopic catalog}

\subsection{Member selection}

Out of 93 galaxies having redshifts, the identification of cluster members proceeds in two steps, following a procedure already used for nearby and medium-redshift clusters (Fadda et al. 1996; Girardi et al. 1996; Girardi \& Mezzetti 2001).

First, we perform the cluster-member selection in velocity space by using only redshift information. We apply the adaptivekernel method (Pisani 1993) to find the significant (>99\% c.1.) peaks in the velocity distribution. This procedure detects A697 


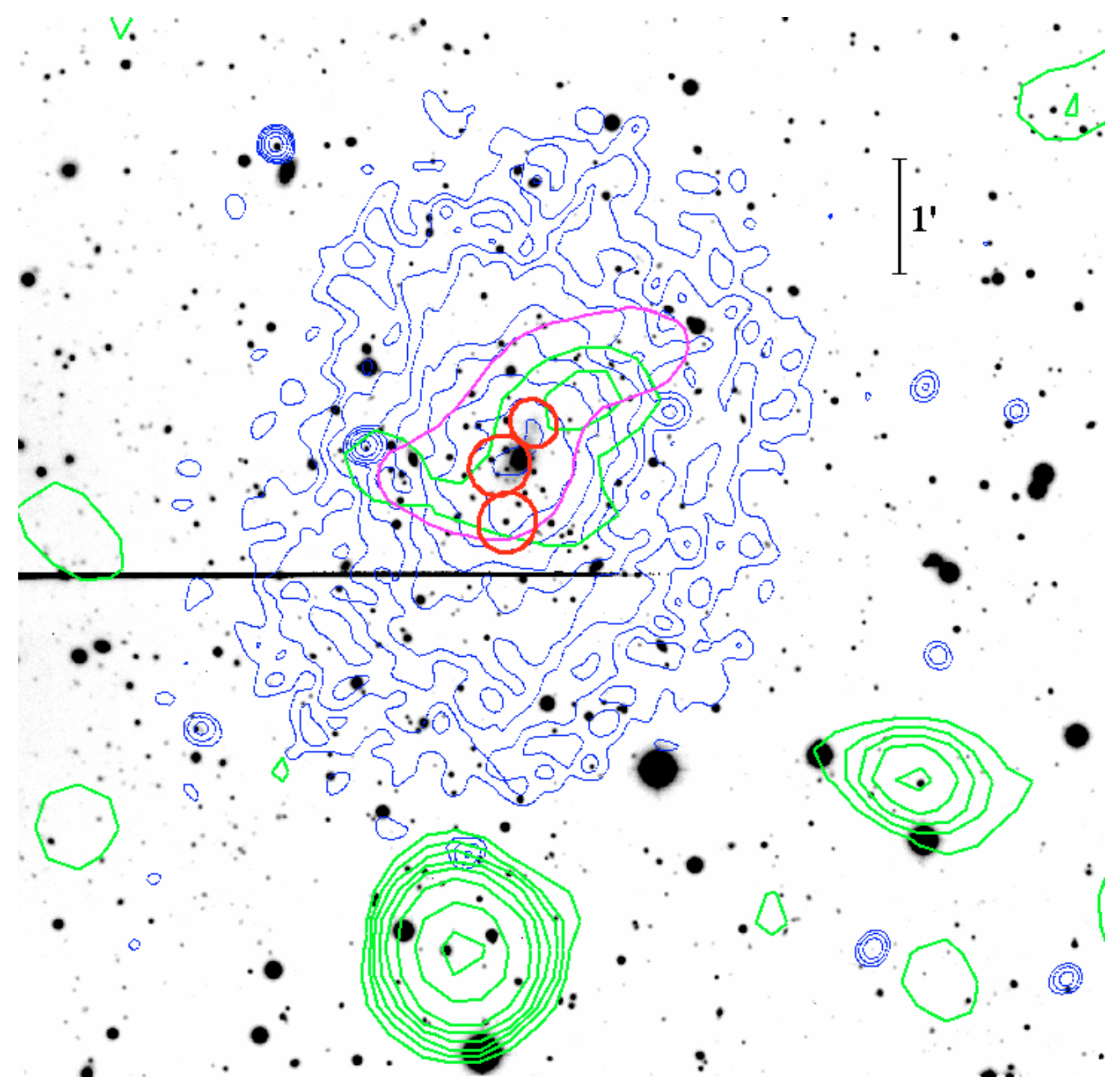

Fig. 2. $R$-band image of the cluster A697 with, superimposed, the contour levels of the Chandra X-ray image ID \#4217 (blue, photons in the energy range 0.3-7 keV) and NVSS (Condon et al. 1998) radio image (green, see also Kempner \& Sarazin 2001). Red ellipses identify structures detected by Wavdetect. To avoid confusion, only one isodensity contour of the spatial distribution of the (likely) cluster members is shown (magenta, see also Fig. 9). North is at the top and East to the left.

as a well isolated peak at $\langle z\rangle=0.282$ assigning 79 galaxies considered as candidate cluster members (see Fig. 3). Out of nonmember galaxies, six and eight are foreground and background galaxies, respectively.

All the galaxies assigned to the A697 peak are analyzed in the second step, which uses a combination of position and velocity information. We apply the procedure of the "shifting gapper" by Fadda et al. (1996). This procedure rejects galaxies that are too far in velocity from the main body of galaxies and within a fixed bin that shifts along the distance from the cluster center. The procedure is iterated until the number of cluster members converges to a stable value. Following Fadda et al. (1996) we use a gap of $1000 \mathrm{~km} \mathrm{~s}^{-1}$ in the cluster restframe and a bin of $0.6 h_{70}^{-1} \mathrm{Mpc}$, or large enough to include 15 galaxies. As for the cluster center, we consider the position of the cD galaxy $\left[\mathrm{RA}=08^{\mathrm{h}} 42^{\mathrm{m}} 57.55\right.$, Dec $=+36^{\circ} 21^{\prime} 59^{\prime} \cdot 9$ (J2000.0)]. The shifting-gapper procedure rejects eight galaxies as non-members (cross symbols in Fig. 4). Following Girardi \& Mezzetti (2001) we also reject three emission line galaxies: two of them dist $\sim 4000 \mathrm{~km} \mathrm{~s}^{-1}$ from the mean velocity in the cluster rest frame, the third has an uncertain redshift since determined by only one emission line.

The member selection procedure leads to a sample of 68 cluster members (see Table 1, Figs. 1 and 4).

The above member selection was planned to be suitable for a single galaxy system. Since the inspection of the velocity distribution of A697 (see also Sect. 3.4) suggests the possible presence of many structures, we also consider the following alternative member selection. We select all (non emission-line) galaxies in the range $v \in\left[81888 \mathrm{~km} \mathrm{~s}^{-1}, 87994 \mathrm{~km} \mathrm{~s}^{-1}\right.$ ], thus to consider 


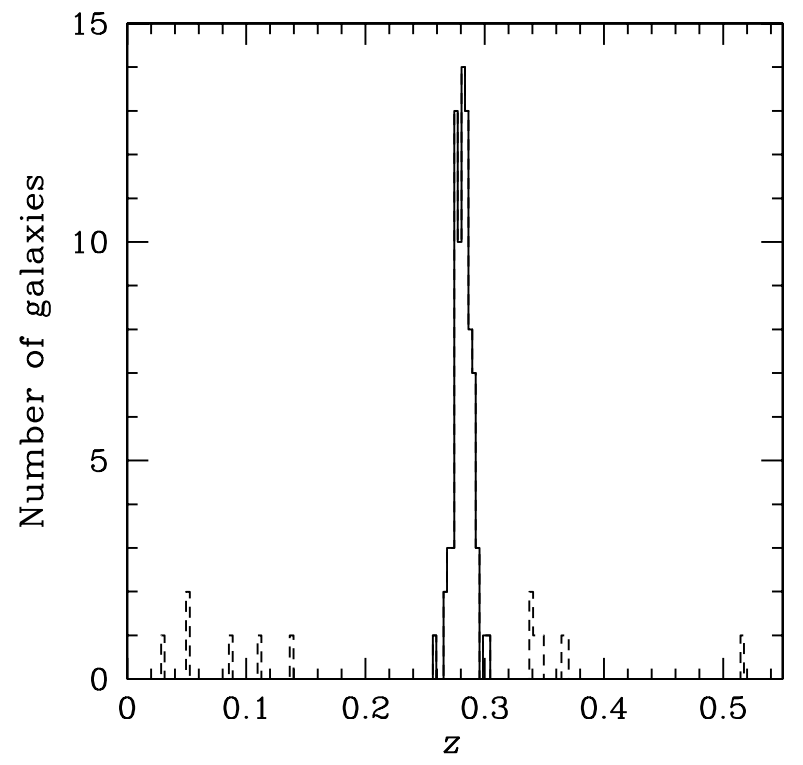

Fig. 3. Redshift galaxy distribution. The solid-line histogram refers to galaxies assigned to the cluster peak according to the adaptive-kernel reconstruction method.

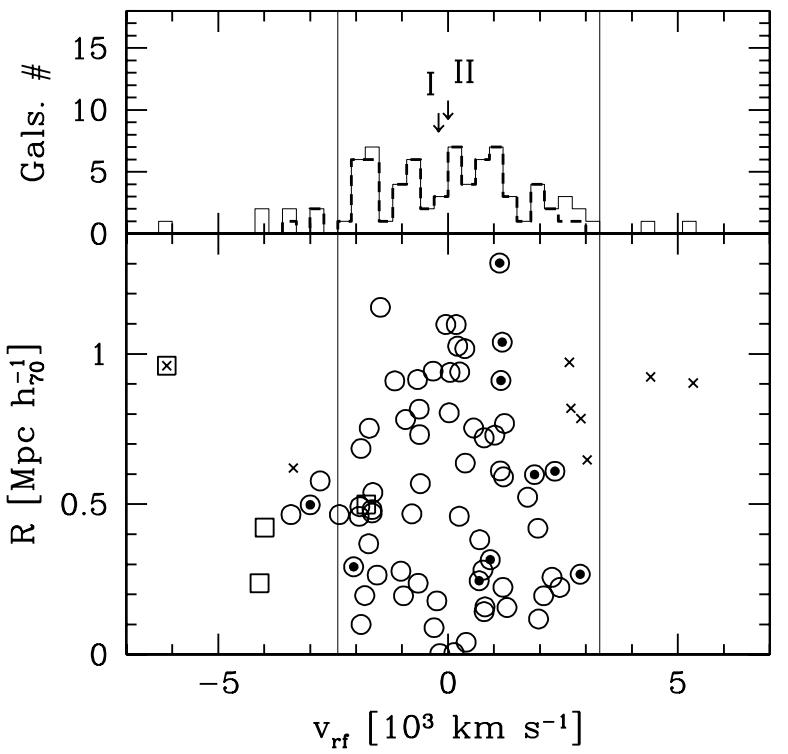

Fig. 4. Lower panel: rest-frame velocity vs. projected clustercentric distance for the 79 galaxies in the main peak (Fig. 3), where we indicate galaxies rejected by the "shifting gapper" method (crosses) and emission line galaxies (squares). The remaining 68 fiducial cluster-members (sample-A) are indicated by open circles (out of which blue galaxies are indicated by a small solid circle, see Sect. 3.3). Upper panel: velocity distribution of all 79 galaxies in the main peak (faint solid-line) and 68 member galaxies (dashed line). Velocities of the main and secondary nuclei of the cD galaxy are pointed out (IDs 41 and 42, respectively). In both panels faint vertical lines indicate velocity boundaries of the alternative sample of member galaxies (sample-B, see text).

all the apparent overdensities in the velocity distribution and reject the underpopulated tails. We obtain a sample of 69 galaxies. When necessary to avoid confusion, we refer to this alternative sample as sample-B, and to the main one as sample-A. The superposition between the two samples is very large, concerning 65 galaxies. If not explicitly said, our analyses always refers to the main sample-A.

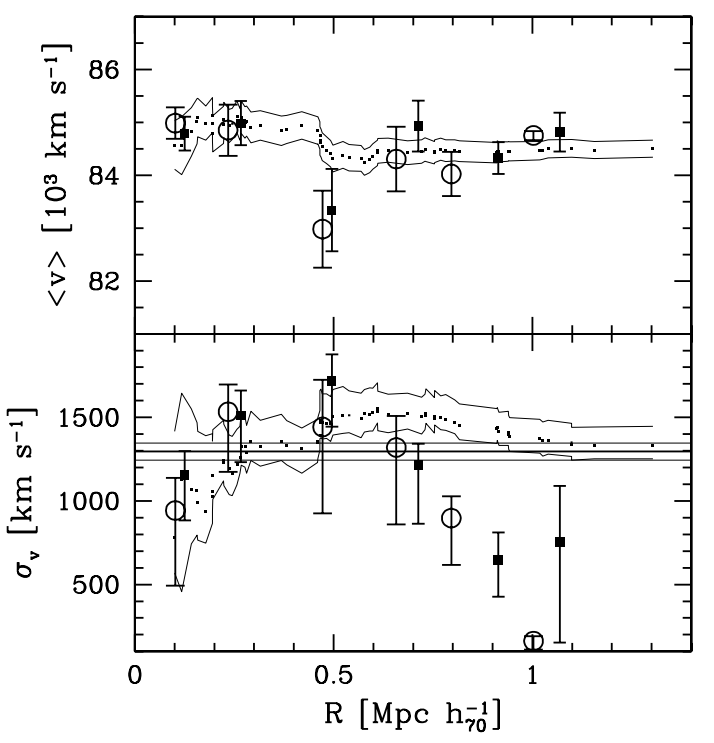

Fig. 5. Differential (solid squares) and integral (small points) profiles of mean velocity (upper panel) and LOS velocity dispersion (lower panel). As for the differential profiles, results for six annuli from the cluster center, each of $0.2 h_{70}^{-1} \mathrm{Mpc}$, are shown. As for the integral profiles, the mean and dispersion at a given (projected) radius from the cluster center is estimated by considering all galaxies within that radius - the first point is obtained on the basis of the five galaxies close to the cluster center. In the lower panel, the horizontal line represents the X-ray temperature with the respective 68 per cent errors transformed in $\sigma_{v}$ assuming the density-energy equipartition between gas and galaxies, i.e. $\beta_{\text {spec }}=1$ (see text). In both panels differential profiles for red galaxies is also shown (open circles).

\subsection{Global properties}

By applying the biweight estimator to cluster members (Beers et al. 1990), we compute a mean cluster redshift of $\langle z\rangle=$ $0.2815 \pm 0.0005$, i.e. $\langle v\rangle=(84505 \pm 163) \mathrm{km} \mathrm{s}^{-1}$. We estimate the LOS velocity dispersion, $\sigma_{v}$, by using the biweight estimator and applying the cosmological correction and the standard correction for velocity errors (Danese et al. 1980). We obtain $\sigma_{v}=1334_{-95}^{+114} \mathrm{~km} \mathrm{~s}^{-1}$, where errors are estimated through a bootstrap technique.

To evaluate the robustness of the $\sigma_{v}$ estimate we analyze the velocity dispersion profile (see Fig. 5). The integral profile smoothly decreases and then flattens in external cluster regions (at $\sim 1 h_{70}^{-1} \mathrm{Mpc}$ ) suggesting that a robust value of $\sigma_{v}$ is asymptotically reached, as found for most nearby clusters (e.g., Fadda et al. 1996; Girardi et al. 1996). Both integral and differential velocity dispersion profiles sharply rise in the central region out to $\sim 0.5 h_{70}^{-1} \mathrm{Mpc}$. This behaviour might be a signature of a relaxed cluster due to circular velocities and galaxy mergers phenomena in the central cluster region (e.g., Merritt 1987; Merritt 1988; Menci \& Fusco Femiano 1996; Girardi et al. 1998) or, alternatively, the consequence of the presence of subclumps, having different mean velocities, LOS aligned in the central region.

In the next sections we further analyze the internal structure of A697 following two alternative possibilities: a) the presence of velocity and spatial segregation of galaxies with respect to their colours and luminosities, which is often taken as evidence of advanced dynamical evolution of the parent cluster (Sect. 3.3); b) the presence of substructures which is indicative of a cluster still far from a complete dynamical relaxation (Sects. 3.4 and 3.5). 
Here we assume that A697 is in dynamical equilibrium to compute virial global quantities. Following the prescriptions of Girardi \& Mezzetti (2001), we assume for the radius of the quasi-virialized region $R_{\mathrm{vir}}=0.17 \times \sigma_{v} / H(z)=3.85 h_{70}^{-1} \mathrm{Mpc}-$ see their Eq. (1) after introducing the scaling with $H(z)$ (see also Eq. (8) of Carlberg et al. 1997 for $R_{200}$ ). Therefore, we have redshifts for galaxies out to a radius of $R_{\text {out }} \sim 5^{\prime} \sim$ $0.34 \times R_{\text {vir }}$ from the cluster center and we sample the region within $R_{\max } \sim 3^{\prime} \sim 0.2 \times R_{\text {vir }}$.

One can compute the mass using the virial theorem (Limber \& Mathews 1960; see also, e.g., Girardi et al. 1998) under the assumption that mass follows galaxy distribution and using the data for the $N_{\mathrm{g}}$ observed galaxies:

$M=M_{\mathrm{svir}}-\mathrm{SPT}$,

where

$M_{\mathrm{svir}}=3 \pi / 2 \cdot \sigma_{v}^{2} R_{\mathrm{PV}} / G$

is the standard virial mass and SPT is the surface pressure term correction (The \& White 1986). The size $R_{\mathrm{PV}}$, equal to two times the (projected) harmonic radius, is:

$R_{\mathrm{PV}}=N_{\mathrm{g}}\left(N_{\mathrm{g}-1}\right) /\left(\sum_{i>j} R_{i j}^{-1}\right)$,

where $R_{\mathrm{ij}}$ is the projected distance between two galaxies.

The estimate of $\sigma_{v}$ is generally robust when computed within a large cluster region (see Fig. 5 for A697 and Fadda et al. 1996 for other examples) and thus we consider our global value. The value of $R_{\mathrm{PV}}$ depends on the size of the sampled region and possibly on the quality of the spatial sampling (e.g., whether the cluster is uniformly sampled or not). Here we consider the well sampled region within $R_{\max }$ obtaining $R_{\mathrm{PV}}=(0.75 \pm$ 0.06) $h_{70}^{-1} \mathrm{Mpc}$, where the error is obtained via a jacknife procedure. The value of SPT correction strongly depends on the amount of the radial component of the velocity dispersion at the radius of the considered region and could be obtained by analyzing the velocity-dispersion profile, although this procedure would require several hundreds of galaxies. Combining data on many clusters one obtains that velocities are isotropic and that the SPT correction at $\sim R_{\text {vir }}$ is SPT $=0.2 \cdot M_{\text {svir }}$ (e.g., Carlberg et al. 1997; Girardi et al. 1998). Due to the limited extension of our sample we prefer to recompute the SPT correction using Eq. (8) of Girardi et al. (1998) and assuming a galaxy King-like distribution with parameters typical of nearby/medium-redshift clusters: a core radius $R_{\mathrm{c}}=1 / 20 \times R_{\mathrm{vir}}$ and a slope-parameter $\beta_{\text {fit }}=0.8$, i.e. the volume galaxy density at large radii as $r^{-3 \beta_{\text {fit }}}=$ $r^{-2.4}$ (Girardi \& Mezzetti 2001). We obtain SPT $=0.35 \cdot M_{\text {svir }}$ and $M\left(<R_{\max }=0.75 h_{70}^{-1} \mathrm{Mpc}\right)=9.5_{-1.5}^{+1.8} \times 10^{14} h_{70}^{-1} M_{\odot}$.

Calling into question the quality of the spatial sampling, one could use an alternative estimate of $R_{\mathrm{PV}}$ on the basis of the knowledge of the galaxy distribution (see Eq. (13) of Girardi et al. 1998). We obtain $R_{\mathrm{PV}}=0.73 h_{70}^{-1} \mathrm{Mpc}$ where a $25 \%$ error is expected due to the fact that typical, rather than individual, galaxy distribution parameters are assumed. This leads to a mass of $M\left(<R_{\max }=0.75 h_{70}^{-1} \mathrm{Mpc}\right)=9.3_{-2.7}^{+2.8} \times 10^{14} h_{70}^{-1} M_{\odot}$, in good agreement with our first estimate.

We can use the second of the above approaches to obtain the mass within the whole assumed virialized region, which is larger than that sampled by observations $M\left(<R_{\text {vir }}=3.85 h_{70}^{-1} \mathrm{Mpc}\right)=$ $4.5_{-1.3}^{+1.4} \times 10^{15} h_{70}^{-1} M_{\odot}$, where we use a $20 \%$ SPT-correction, suitable in this case.

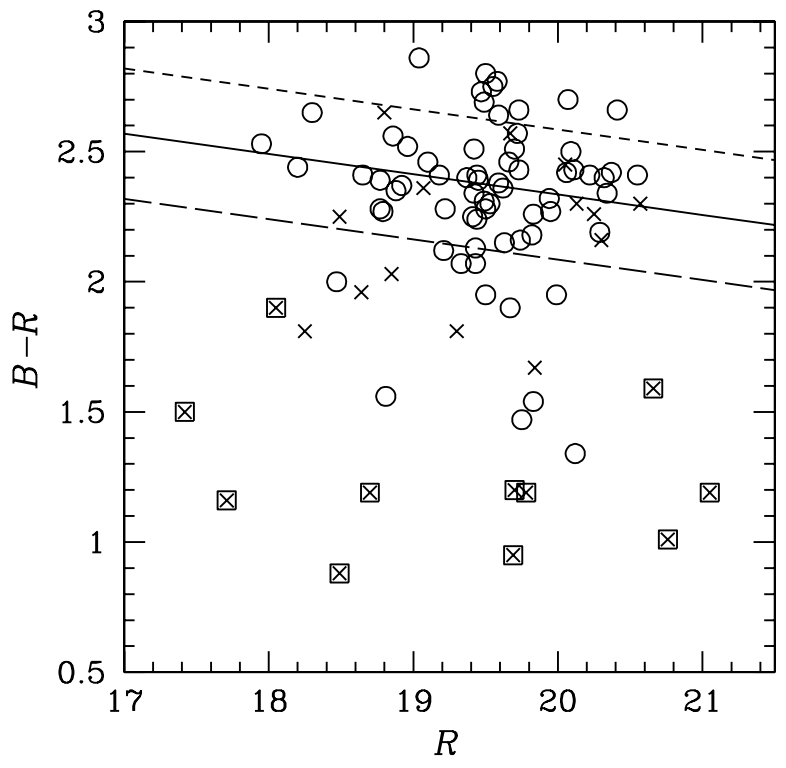

Fig. 6. $B-R$ vs. $R$ diagram for galaxies with available spectroscopy: circles and crosses denote cluster and field members, respectively. Out of field members, squares denote emission line galaxies. The solid line gives the best-fit colour-magnitude relation; the dashed lines are drawn at $\pm 0.25 \mathrm{mag}$ from the CMR. According to our working definition in Sect. 3.3 cluster members are divided into blue and red galaxies (below and above the long-dashed line, respectively). In particular, very/not very red galaxies lie above/below the solid line.

\subsection{Testing galaxy segregation}

The presence of velocity and spatial segregation of galaxies with respect to their colours, luminosities and morphologies is often taken as evidence of advanced dynamical evolution of the parent cluster (e.g., Biviano et al. 1992; Ellingson et al. 2001; Biviano et al. 2002; Poggianti 2004; Goto 2005). Here we check for possible luminosity and colour segregation of galaxies, both in position and in velocity space, by using the sample of 67 galaxies to which we have successfully assigned $R$ magnitude and $B-R$ colours out of the 68 member galaxies.

As for luminosity segregation, we find no significant correlation between the absolute velocity $|v|$ and $R$ magnitude, as well as between clustercentric distance $R$ and $R$ magnitude. We also divide the sample into low- and high-luminosity subsamples by using the median $R$ magnitude $=19.5$. To test for different means and variances in velocity distributions of less- and moreluminous galaxies, we apply the standard means-test and F-test (e.g., Press et al. 1992). We obtain no significant difference. Moreover, we verify that the two subsamples do not have different distributions of $v, R, 2 \mathrm{D}$ spatial positions, and $[v, R]$ using the 1D and 2D Kolmogorov-Smirnov tests (hereafter 1DKS and 2DKS-tests. See, e.g., Ledermann 1982 for 1DKS; see Fasano \& Franceschini 1987 for 2DKS, as implemented by Press et al. 1992).

As for colour segregation, we find only a very marginal anticorrelation between the clustercentric distance $R$ and $B-R$ colour (at the $93.27 \%$ c.l., according to the Kendall non-parametric rank correlation test). We also use the colour-magnitude relation (hereafter CMR), which indicates the early-type galaxy locus, to divide the sample into some subsets and then compare their properties. To determine CMR we fix the slope according to López-Cruz et al. (2004, see their Fig. 3) and apply the twosigma-clipping fitting procedure to the cluster members obtaining $B-R=3.895-0.0780 \times R$ (see Fig. 6). "Blue" objects 
Table 2. Results of kinematical analysis.

\begin{tabular}{lccc}
\hline \hline Sample & $N_{\mathrm{g}}$ & $\begin{array}{c}\langle v\rangle \\
\mathrm{km} \mathrm{s}^{-1}\end{array}$ & $\begin{array}{c}\sigma_{v}^{a} \\
\mathrm{~km} \mathrm{~s}^{-1}\end{array}$ \\
\hline Whole system (sample A) & 68 & $84505 \pm 163$ & $1334_{-95}^{+114}$ \\
Blue galaxies & 11 & $85954 \pm 487$ & $1509_{-507}^{+763}$ \\
Red galaxies & 56 & $84288 \pm 167$ & $1243_{-85}^{+120}$ \\
Whole system (sample B) & 69 & $84790 \pm 163$ & $1349_{-63}^{+112}$ \\
Sample A without KMM4g3 gals & 61 & $84296 \pm 174$ & $1353_{-97}^{+122}$ \\
\hline
\end{tabular}

$a$ We use the biweight and the gapper estimators by Beers et al. (1990) for samples with $N_{\mathrm{g}} \geq 15$ and with $N_{\mathrm{g}}<15$ galaxies, respectively (see also Girardi et al. 1993).

are defined to be those galaxies at least 0.25 mag bluer in $B-R$ than the colour of the CMR. These blue galaxies have typically $B-R \lessgtr 2$, thus include spiral galaxies and exclude elliptical galaxies, according to the expected typical colours at the cluster redshift (Buta et al. 1994; Buta \& Williams 1995; Poggianti 1997). Thus, the remaining objects, which we define as the "red" sample, should consist mostly of ellipticals and lenticulars. We also divide red galaxies in "very red" and "not very red" depending on whether they lie above or below the CMR. The blue sample (marginally) differs from the red sample in the 2D galaxyposition distribution, the distribution in the $[v, R]$ plane and the velocity distribution (at the $93.40 \%, 94.20 \%$ and $94.45 \%$ c.l.s., according to the KS-tests, respectively). Instead, no difference is found between very red and not very red galaxies.

Table 2 shows $\langle v\rangle$ and $\sigma_{v}$ estimates for blue and red galaxies: the $\langle v\rangle$ of blue galaxies is higher than that of red galaxies at the $97.37 \%$ c.l., according to the means-test. The difference we detect in the mean velocity suggests the existence of a high velocity group, mainly populated by late-type galaxies.

\subsection{Analysis of substructure}

We analyze the velocity distribution to look for possible deviations from Gaussianity that could provide important signatures of complex dynamics. For the following tests the null hypothesis is that the velocity distribution is a single Gaussian.

We estimate three shape estimators, i.e. the kurtosis, the skewness, and the scaled tail index (see, e.g., Beers et al. 1991). The value of the normalized kurtosis $(-0.71)$ and of the scaled tail index (0.839) shows evidence that the velocity distribution differs from a Gaussian, being lighter-tailed, with a c.l. of 90-95\% (see Table 2 of Bird \& Beers 1993).

Then, we investigate the presence of gaps in the distribution using the ROSTAT package (Beers et al. 1990). A weighted gap in the space of the ordered velocities is defined as the difference between two contiguous velocities, weighted by the location of these velocities. The weight is $i \times\left(N_{\mathrm{g}}-i\right)$, where $i$ gives the location of the object preceding the gap and $N_{\mathrm{g}}$ is the total number of galaxies. We obtain values for these gaps relative to their average size, precisely the midmean of the weighted-gap distribution. We look for normalized gaps larger than 2.25 since in random draws of a Gaussian distribution they arise at most in about 3\% of the cases, independent of the sample size (Wainer \& Schacht 1978; see also Beers et al. 1991). Three significant gaps in the ordered velocity dataset are detected (see Fig. 7). In Table 3 we list the number of galaxies and the velocity of the object preceding the gap, the normalized size (i.e., the "importance") of the gap itself, and the probability of finding a normalized gap of this size

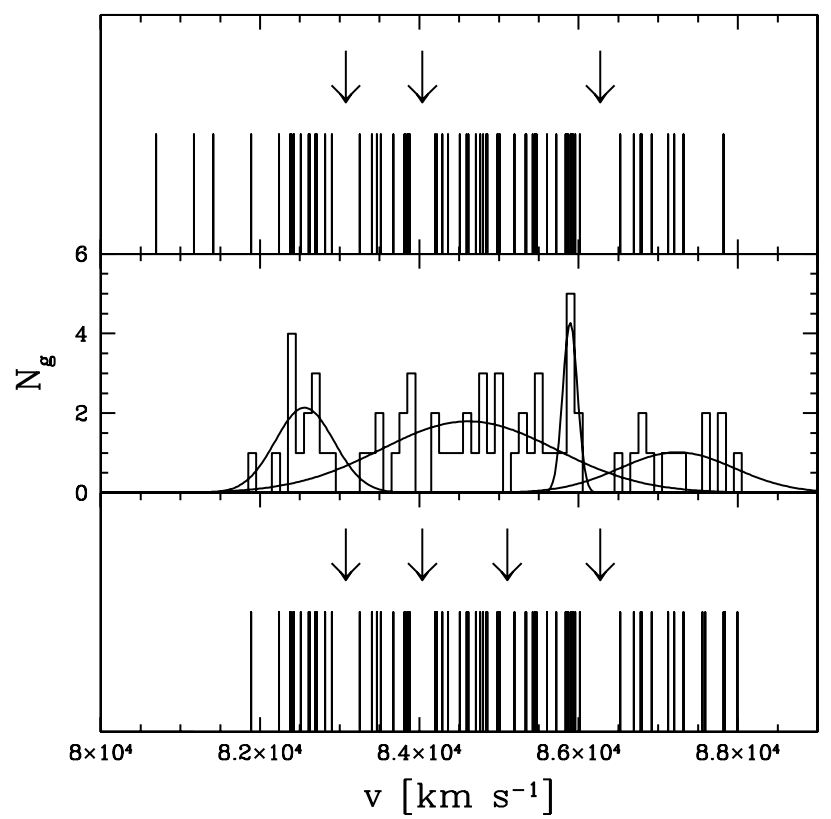

Fig. 7. Velocity distribution of radial velocities for the cluster members. Upper and lower panels: stripe density plots where arrow indicate the position of the significant gaps (samples-A and B, respectively). Central panel: velocity histogram with a binning of $100 \mathrm{~km} \mathrm{~s}^{-1}$ with the Gaussians corresponding to the most significant KMM partition for the sample-B.

Table 3. Results of weighted-gap analysis.

\begin{tabular}{lcccc}
\hline \hline Sample & $N_{\text {galsprec }}$ & $\begin{array}{c}v_{\text {prec }} \\
\mathrm{km} \mathrm{s}^{-1}\end{array}$ & Size & Prob. \\
\hline Sample A) & 17 & 82900 & 2.73 & $1.4 \mathrm{E}-2$ \\
Sample A) & 27 & 83882 & 2.91 & $6.0 \mathrm{E}-3$ \\
Sample A) & 32 & 86018 & 2.56 & $1.4 \mathrm{E}-2$ \\
Sample B) & 14 & 82900 & 2.62 & $1.4 \mathrm{E}-2$ \\
Sample B) & 24 & 83882 & 2.93 & $6.0 \mathrm{E}-3$ \\
Sample B) & 39 & 85011 & 2.33 & $3.0 \mathrm{E}-2$ \\
Sample B) & 56 & 86018 & 3.05 & $2.0 \mathrm{E}-3$ \\
\hline
\end{tabular}

and with the same position in a normal distribution (as computed with the ROSTAT package, Beers et al. 1990). Using the results of our weighted-gap analysis, we divide the dataset in four subsets containing 17, 10, 32 and 9 galaxies from low to high velocities. We compare these subsets two by two by applying the 2DKS-test to the galaxy positions. We find no difference.

The velocity of the $\mathrm{cD}$ galaxy (main nucleus, $v=$ $84361 \mathrm{~km} \mathrm{~s}^{-1}$ ) shows no evidence of peculiarity according to the Indicator test by Gebhardt \& Beers (1991).

The cluster velocity field may be influenced by the presence of internal substructures. To investigate the velocity field of A697, we divide galaxies into low- and high-velocity subsamples by using the median value of galaxy velocities $\bar{v}=$ $84664 \mathrm{~km} \mathrm{~s}^{-1}$, and check the difference between the spatial distributions of the two samples. We find no difference between high- and low-velocity galaxies. We also perform a multiple linear regression fit to the observed velocities with respect to the galaxy positions in the plane of the sky (see also Girardi et al. 1996; den Hartog \& Katgert 1996), but we do not find any significant velocity gradient.

We combine galaxy velocity and position information to compute the $\Delta$-statistics devised by Dressler \& Schectman (1988). This test is sensitive to spatially compact subsystems 
that have either an average velocity that differs from the cluster mean, or a velocity dispersion that differs from the global one, or both. We find $\Delta=69$ for the value of the parameter which gives the cumulative deviation. This value is not a significant indication of substructure as assessed computing 1000 Monte Carlo simulations, randomly shuffling the galaxy velocities.

Different substructure-tests are sensitive to different kinds of substructure (Pinkney et al. 1996). Since above we find evidence of substructure only from 1D tests we think that the possible subclumps are (almost) LOS aligned. Therefore, we attempt to detect subsets in the velocity distribution resorting to the Kaye's mixture model (KMM) test as implemented by Ashman et al. (1994). The KMM algorithm fits a user-specified number of Gaussian distributions to a dataset and assesses the improvement of that fit over a single Gaussian. In addition, it provides the maximum-likelihood estimate of the unknown $n$-mode Gaussians and an assignment of objects into groups. KMM is most appropriate in situations where theoretical and/or empirical arguments indicate that a Gaussian model is reasonable. The Gaussian is valid in the case of cluster velocity distributions, where gravitational interactions drive the system toward a relaxed configuration with a Gaussian velocity distribution. However, one of the major uncertainties of this method is the optimal choice of the number of groups for the partition. We use the results of the gap analysis to determine the first guess for the group partition and we try to fit two, three or four velocity groups. We do not find any group partition that is a significantly better descriptor of the velocity distribution than a single Gaussian.

\subsection{Further insights into substructure}

In this section we analyze the alternative sample of cluster members (sample-B), which is likely more suitable to represent a multi-Gaussians system.

The analysis of the alternative sample leads to similar global properties, i.e. $\langle v\rangle=(84790 \pm 163) \mathrm{km} \mathrm{s}^{-1}, \sigma_{v}=$ $1349_{-63}^{+112} \mathrm{~km} \mathrm{~s}^{-1}, M\left(<0.75 h_{70}^{-1} \mathrm{Mpc}\right)=9.7_{-1.2}^{+1.8} \times 10^{14} h_{70}^{-1} M_{\odot}$, and $M\left(<R_{\text {vir }}=3.89 h_{70}^{-1} \mathrm{Mpc}\right)=4.6_{-1.2}^{+1.4} \times 10^{15} h_{70}^{-1} M_{\odot}$. We also obtain similar results in the substructure analysis in the sense that only velocity distribution shows signs of possible substructure. In particular, the velocity distribution shows a departure from Gaussian at the $98 \%-99 \%$ c.l. according to the W-test (Shapiro \& Wilk 1965) and the value of kurtosis (-0.92). We also find an additional significant weighted gap with respect to sample-A (see Fig. 7). This additional gap is due to the fact that the slight change of the sample (and of the consequent values of the weights, see Sect. 3.4) slightly enhances the size of this gap from 2.21 to 2.33 , i.e. beyond our threshold of 2.25 . In addition, the velocity of the cD galaxy shows marginal evidence of peculiarity according to the Indicator test by Gebhardt \& Beers (1991, at the $90-95 \%$ c.1.).

However, while in the sample-A the KMM analysis fails to detect a significant partition, in the sample-B we find that each of the $2 / 3 / 4 / 5$ group partitions is a better descriptor of the velocity distribution at the $99.5 \% / 98.8 \% / 99.6 \% / 99.3 \%$ c.ls., according to the likelihood ratio test. The KMM results differ so much between the sample-A and B that we investigated the possible cause. The difference is likely due to the three lowest-velocity galaxies in the sample-A: after rejecting these three galaxies, the KMM analysis of sample-A gave similar results to those of sample-B.
Table 4. Kinematical analysis for KMM groups.

\begin{tabular}{lrcr}
\hline \hline Sample & \multicolumn{1}{c}{$N_{\mathrm{g}}$} & $\begin{array}{c}\langle v\rangle \\
\mathrm{km} \mathrm{s}^{-1}\end{array}$ & \multicolumn{1}{c}{$\begin{array}{c}\sigma_{v}^{a} \\
\mathrm{~km} \mathrm{~s}^{-1}\end{array}$} \\
\hline KMM2g1 & 12 & $82545 \pm 41$ & $134_{-38}^{+20}$ \\
KMM2g2 & 57 & $85273 \pm 148$ & $1110_{-91}^{+99}$ \\
\hline KMM3g1 & 12 & $82545 \pm 41$ & $134_{-38}^{+20}$ \\
KMM3g2 & 49 & $84951 \pm 126$ & $875_{-75}^{+101}$ \\
KMM3g3 & 8 & $87557 \pm 104$ & $260_{-30}^{+54}$ \\
\hline KMM4g1 & 14 & $82559 \pm 56$ & $198_{-42}^{+54}$ \\
KMM4g2 & 35 & $84616 \pm 104$ & $605_{-43}^{+64}$ \\
KMM4g3 $^{b}$ & 7 & $85894 \pm 19$ & $51_{-82}^{+82}$ \\
KMM4g4 $^{13}$ & $87238 \pm 116$ & $394_{-41}^{+69}$ \\
\hline KMM5g1 $^{14}$ & $82559 \pm 56$ & $198_{-42}^{+54}$ \\
KMM5g2 $^{10}$ & $83682 \pm 60$ & $176_{-12}^{+68}$ \\
KMM5g3 $^{24}$ & $84949 \pm 77$ & $336_{-28}^{+42}$ \\
KMM5g4 $^{b}$ & 8 & $85907 \pm 19$ & $51_{-82}^{+82}$ \\
KMM5g5 $^{13}$ & $87238 \pm 116$ & $394_{-41}^{+69}$ \\
\hline
\end{tabular}

a We use the biweigth and the gapper estimators by Beers et al. (1990) for samples with $N_{\mathrm{g}} \geq 15$ and with $N_{\mathrm{g}}<15$ galaxies, respectively (see also Girardi et al. 1993).

$b$ We list the value of $\sigma_{v}$ before the correction for the estimated errors in the redshift measures since the correction leads to a negative value.

For each out of the $2 / 3 / 4 / 5$ group partitions we use the KMM code to assign members to the respective groups. Table 4 lists the results for the kinematical analysis. Note that the uncertainty in KMM membership assignments leads to an artificial truncation of the tails of the distributions and this may leading to lower estimates for the velocity dispersion (Bird 1994). Figure 7 shows the four group partition (hereafter KMM4g1, KMM4g2, KMM4g3, KMM4g4), which is the most significant one.

Looking for correlations between positions and velocities, we compare the KMM groups two by two by applying the 2DKS-test to the galaxy position of member galaxies. In the four-group partition we obtain a marginal difference when comparing KMM4g3 with KMM4g1(KMM4g2) at the 95.24\%(91.12\%) c.1.

The KMM4g3 group (gals. with IDs. 4, 7, 16, 27, 31, 84, 93) has a very small velocity dispersion, and shows a very sparse 2D distribution with respect to the rest of the cluster. Moreover, out of the six KMM4g3 galaxies having $B-R$ colours, half of them belong to the blue sample according to our definition in Sect. 3.3. Therefore, this group might be related to the high velocity group suggested by our analysis in Sect. 3.3.

\section{2D galaxy distribution}

By applying the $2 \mathrm{D}$ adaptive-kernel method to the position of A697 galaxy members we find only one peak. However, our spectroscopic data do not cover the entire cluster field and suffer from magnitude incompleteness. To overcome these limits we recover our photometric catalog. We consider galaxies (objects with SExtractor stellar index $\leq 0.9$ ) lying within 0.25 mag of the CMR determined in Sect. 3.3. The inspection of Fig. 6 suggests some contamination by field galaxies already for $20 \leq R \leq 21$ (cross symbols), thus we do not show results for galaxies fainter 


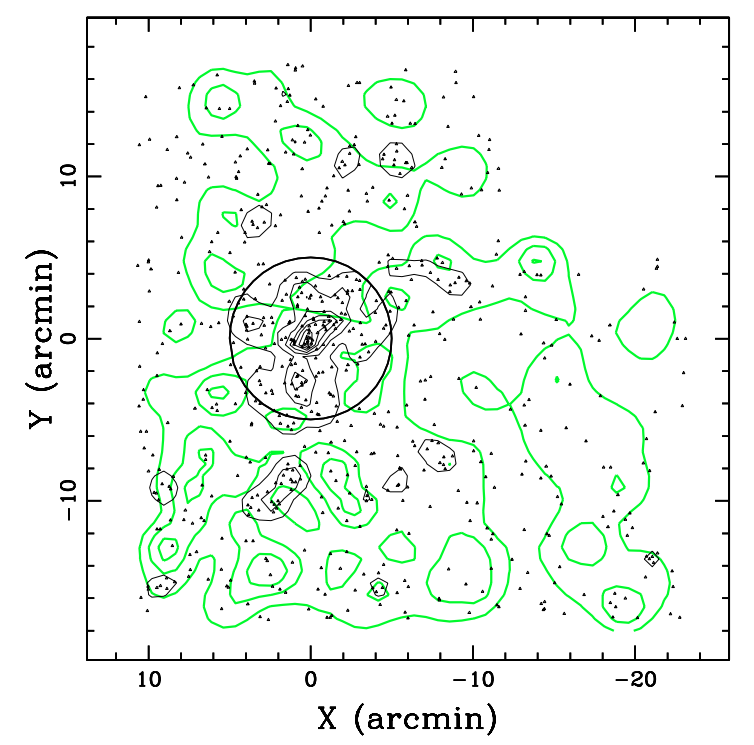

Fig. 8. Spatial distribution on the sky and relative isodensity contour map of 645 likely cluster members (according to the colour-magnitude relation) with $R \leq 21$, obtained with the adaptive-kernel method (black lines). For comparison we show the contour map of the 749 likely noncluster members (grey lines). The plot is centered on the cluster center. The circle indicate the $5^{\prime}$ central region.

than 21 mag. Figure 8 shows the contour map for 645 likely cluster members having $R \leq 21$ : the main structure has "S-shape" centered on the $\mathrm{cD}$ galaxy with a secondary, important NW peak. This central structure is surrounded by few significant peaks within a radius of $5^{\prime}$. Another significant, dense peak in the galaxy distribution lies $\sim 9^{\prime}$ south of the cluster center.

The field of A697 is strongly disturbed by the presence of several luminous stars: this might bias our results about $2 \mathrm{D}$ cluster morphology. To tack this problem we consider the distribution of galaxies having $R \leq 21$ and lying more than 0.75 mag from the colour-magnitude relation, i.e. likely mainly formed by non-member galaxies. Members and non-members have rather different $2 \mathrm{D}$ distributions suggesting that our results about cluster morphology are not affected by the presence of disturbing luminous stars.

To definitely exclude the possibility of a biased cluster morphology we also consider: a) the distribution of 149 likely members within a radius of $1.3 h_{70}^{-1} \mathrm{Mpc}\left(\sim 5^{\prime}\right)$ to avoid a very luminous star $\sim 7^{\prime}$ East and a bad line caused by the gap between the CCD chips of the WFC $\sim 5^{\prime}$ North (see Fig. 9); b) the distribution of 54 likely members within a radius of $0.6 h_{70}^{-1} \mathrm{Mpc}\left(\sim 2.5^{\prime}\right)$ to avoid a few luminous south-western stars (see Fig. 10). The first analysis again finds a southern peak: the six galaxies of our spectroscopic catalog located within a radius of $0.25 h_{70}^{-1} \mathrm{Mpc}$ from its center [RA $=08^{\mathrm{h}} 43^{\mathrm{m}} 01^{\mathrm{s}} .1$, Dec $\left.=+36^{\circ} 19^{\prime} 18^{\prime \prime}(\mathrm{J} 2000.0)\right]$ have a mean velocity comparable to that of the whole system. Both a) and b) analyses confirm the S-shape of the main central structure, but we have too poor statistics to analyze the kinematical properties of $\mathrm{E}$ and NW peaks.

NW peak is induced by the presence of faint galaxies, while brilliant galaxies can be described by a single central peak thus in agreement with the 2D analysis of the spectral catalog. Figure 10 shows the contour maps of the distributions of 32 brilliant $(R \leq 20)$ and 22 faint $(20<R \leq 21)$ galaxies within a radius of $0.6 h_{70}^{-1} \mathrm{Mpc}$ : the respective $2 \mathrm{D}$ distributions are different at the $98.48 \%$ c.l., according to the 2DKS test. Suspecting that these faint galaxies belong to a background,

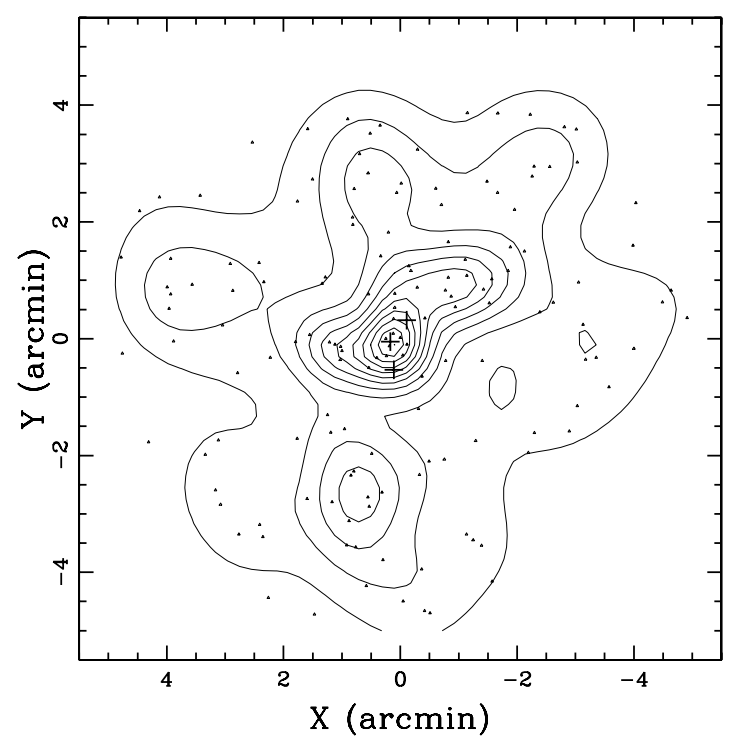

Fig. 9. Spatial distribution on the sky and relative isodensity contour map of 149 likely cluster members with $R \leq 21$ within $1.3 h_{70}^{-1} \mathrm{Mpc}\left(\sim 5^{\prime}\right)$ of the cluster center. The plot is centered on the cluster center. Crosses indicate the position of the centers of the substructures found by the wavelet technique in Chandra X-ray data (see Sect. 5).

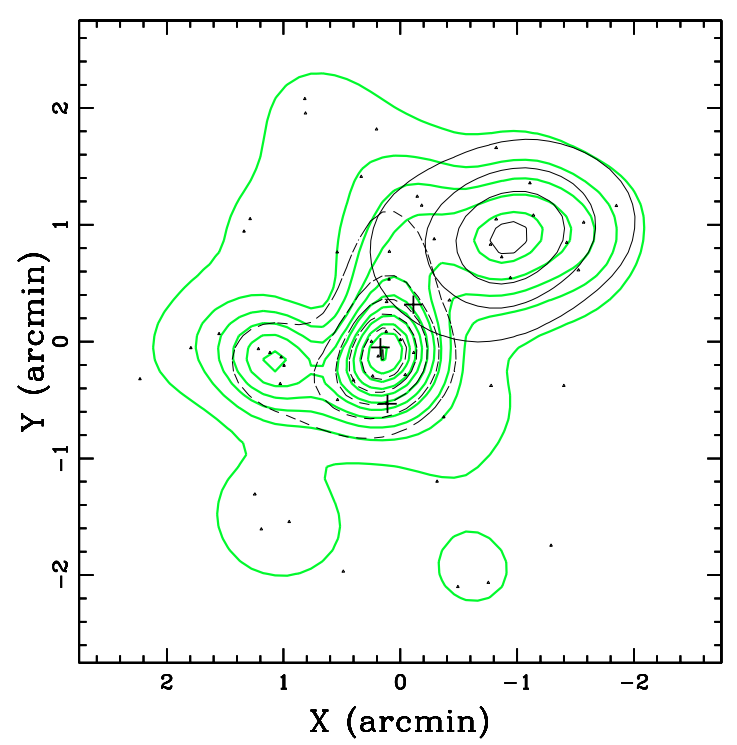

Fig. 10. Spatial distribution on the sky and relative isodensity contour map of 54 likely cluster members with $R \leq 21$ within $0.6 h_{70}^{-1} \mathrm{Mpc}$ of the cluster center (gray lines). The contour maps of brilliant and faint galaxies - i.e. galaxies having $R \leq 20$ and $20<R \leq 21$ - are also shown (black dashed and solid lines, respectively). The plot is centered on the cluster center. Crosses indicate the position of the centers of the substructures found with the wavelet technique in Chandra X-ray data (see Sect. 5).

dense cluster we have studied the corresponding galaxies in our spectroscopic sample, i.e. the five non-member galaxies having $20<R \leq 21$ and lying within $0.25 \mathrm{mag}$ of the CMR (see crosses in Fig. 6). These five galaxies are scattered in redshift, $z \in(0.29,0.52)$, and in projected position around the cluster center. Therefore, we have no evidence for a background NW cluster. 


\section{X-ray data and analysis}

The X-ray analysis of A697 is performed on the archival data of the Chandra ACIS-I observation 800373 (exposure ID \#4217, see Fig. 11). The pointing has an exposure time of $19.8 \mathrm{ks}$. Data reduction is performed using the package $\mathrm{CIAO}^{2}$ (Chandra Interactive Analysis of Observations) on the chip I3 (field of view $\left.\sim 8.5^{\prime} \times 8.5^{\prime}\right)$. First, we remove events from the level 2 event list with a status not equal to zero and with grades one, five and seven. Then, we select all events with energy between 0.3 and $10 \mathrm{keV}$. In addition, we clean bad offsets and examine the data, filtering out bad columns and removing times when the count rate exceeds three standard deviations from the mean count rate per $3.3 \mathrm{~s}$ interval. We then clean the I3 chip of flickering pixels, i.e., times where a pixel has events in two sequential $3.3 \mathrm{~s}$ intervals. The resulting exposure time for the reduced data is $19.5 \mathrm{ks}$.

In Fig. 2 we plot an $R$-band image of the cluster with superimposed the X-ray contour levels of the Chandra image. The shape of the cluster appears to be moderately elliptical. By using the CIAO package Sherpa we fit an elliptical 2D Beta model to the X-ray photon distribution to quantify the departure from the spherical shape. The model is defined as follows:

$f(x, y)=f(R)=A /\left[1+\left(R / R_{0}\right)^{2}\right]^{\alpha}$

where the radial coordinate $R$ is defined as $R(x, y)=\left[X^{2}(1-\right.$ $\left.\epsilon)^{2}+Y^{2}\right]^{1 / 2} /(1-\epsilon), X=\left(x-x_{0}\right) \cos \theta+\left(y-y_{0}\right) \sin \theta$ and $Y=\left(y-y_{0}\right) \cos \theta-\left(x-x_{0}\right) \sin \theta$. Here $x$ and $y$ are physical pixel coordinates on chip I3. The best fit centroid position is located on the main body of the $\mathrm{cD}$ galaxy. The best fit core radius, the ellipticity and the position angle are $R_{0}=49.3^{\prime \prime} \pm 3.5^{\prime \prime}$ (i.e. $\left.210 \pm 15 h_{70}^{-1} \mathrm{kpc}\right), \epsilon=0.26 \pm 0.02$ and $\mathrm{PA}=164.4 \pm 2.3$ degrees (measured from North to East), respectively.

To detect possible substructures in A697 we perform a wavelet analysis by running the task CIAO/Wavdetect on chip I3 (for applications of this technique in the literature see, e.g., Slezak et al. 1994; Vikhlinin et al. 1997; Sun et al. 2002; Ferrari et al. 2005). The task was run on different scales to search for substructures with different sizes. The significance threshold $^{3}$ was set at $10^{-6}$. The results are shown in Fig. 2. Thick ellipses represent three significant surface brightness peaks found by Wavdetect in the core of the cluster. The principal one, located at RA $=08^{\mathrm{h}} 42^{\mathrm{m}} 58^{\mathrm{s}} .4$ and Dec $=+36^{\circ} 21^{\prime} 56^{\prime \prime}$, is centered $15^{\prime \prime} \mathrm{S}-\mathrm{SE}$ from the central $\mathrm{cD}$ galaxy. Two more significant peaks are located at RA $=08^{\mathrm{h}} 42^{\mathrm{m}} 58^{\mathrm{s}} .1$ and Dec $=$ $+36^{\circ} 21^{\prime} 27^{\prime \prime}$, and at RA $=08^{\mathrm{h}} 42^{\mathrm{m}} 57^{\mathrm{s}} .0$ and $\mathrm{Dec}=+36^{\circ} 22^{\prime} 18^{\prime \prime}$, respectively. The wavelet technique reveals the complex structure of the dense central cluster region, apparent in the surface brightness distribution only when a very tuned choice of the contour levels is used.

For the spectral analysis of the cluster X-ray photons, we compute a global estimate of the ICM temperature. The temperature is computed from the spectrum of the cluster within a circular aperture of $2^{\prime}$ radius around the cluster center. Fixing the absorbing galactic hydrogen column density at $3.41 \times 10^{20} \mathrm{~cm}^{-2}$, computed from the HI maps by Dickey \& Lockman (1990), we fit a Raymond-Smith (1977) spectrum using the CIAO package Sherpa with $\chi^{2}$ statistics. We find a best fitting temperature of $T_{\mathrm{X}}=(10.2 \pm 0.8) \mathrm{keV}$ and a metal abundance of $0.36 \pm 0.8$ in solar units.

\footnotetext{
${ }^{2}$ CIAO is freely available at http://asc.harvard.edu/ciao/

3 See Sect. 11.1 of the CIAO Detect Manual (software release version 3.2, available at the WWW site http://cxc.harvard.edu/ciao/manuals.html).
}

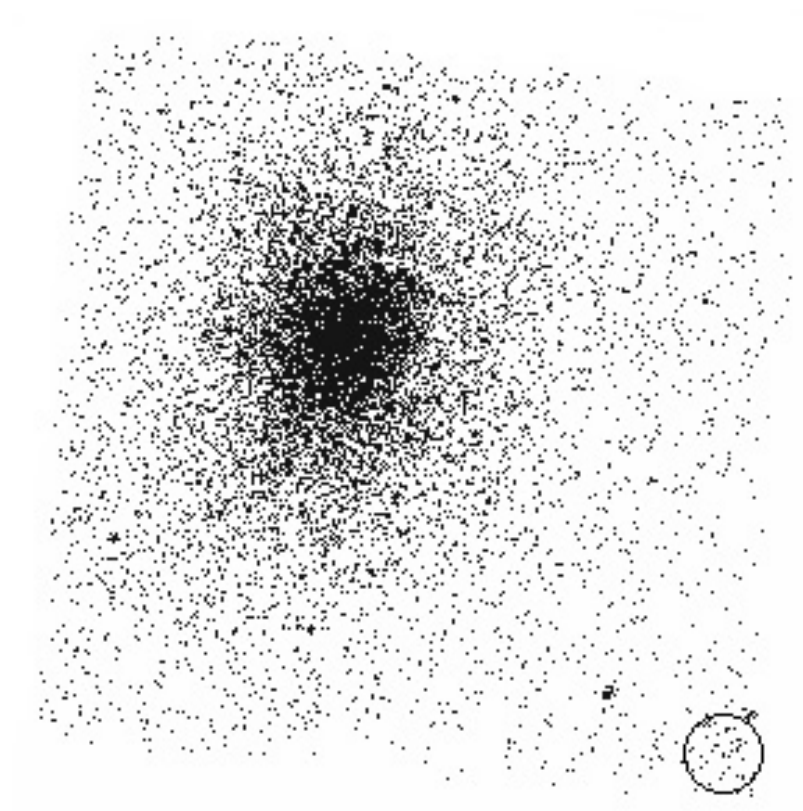

Fig. 11. 8.5' $\times 8.5^{\prime}$ Chandra X-ray image (ID \#4217, chip I3 only) of the cluster A697 in the energy band $0.5-2 \mathrm{keV}$. The circle puts in evidence a faint diffuse emission located $\sim 6^{\prime} \mathrm{SW}$ from the cluster center (see Sect. 5). North is at the top and East to the left.

Very interestingly, the X-ray image (see Fig. 11) also shows the presence of a faint diffuse emission located $\sim 6^{\prime} \mathrm{SW}$ of the cluster center. Already revealed by M00 on a ROSAT HRI image, this source could be an additional infalling group. Unfortunately, we have no way to verify this hypothesis by using redshift data and thus we do not discuss this feature in the following.

\section{Discussion}

We analyze the internal dynamics of A697 on the basis of spectroscopic data for 93 galaxies in a cluster region of a radius of $\sim 5^{\prime}$ (i.e., $\sim 1.3 h_{70}^{-1} \mathrm{Mpc}$ ) from the cD galaxy. We find that A697 appears as a single peak in the redshift space at $\langle z\rangle=0.282$.

Using a standard selection procedure we obtain 68 fiducial cluster members (sample-A). We compute a LOS velocity dispersion of $\sigma_{V}=1334_{-95}^{+114} \mathrm{~km} \mathrm{~s}^{-1}$, higher or comparable to the highest values for other clusters in the literature (see Fadda et al. 1996; Mazure et al. 1996; Girardi \& Mezzetti 2001). We obtain consistent estimates of $\sigma_{V}$ - see Table 2 - when using alternative samples of cluster members, i.e. the sample-B which is likely more suitable for a multipeaked velocity distribution, the red-galaxies sample which is likely less contaminated by field galaxies, and the sample obtained after the rejection of galaxies belonging to the KMM4g3 group (possibly infalling onto the cluster, see Sect. 6.2). Moreover, our estimate of $\sigma_{v}$ is fully consistent with the average X-ray temperature $T_{\mathrm{X}}=10.2 \pm 0.8 \mathrm{keV}$ coming from our analysis of Chandra data when assuming the equipartition of energy density between gas and galaxies (i.e. $\beta_{\text {spec }}=1.06_{-0.17}^{+0.20}$ to be compared with $\beta_{\text {spec }}=1^{4}$, see also Fig. 5).

Assuming that the cluster is in dynamical equilibrium and mass follows galaxy distribution, we compute virial

\footnotetext{
${ }^{4} \beta_{\text {spec }}=\sigma_{v}^{2} /\left(k T / \mu m_{\mathrm{p}}\right)$ with $\mu=0.58$ the mean molecular weight and $m_{\mathrm{p}}$ the proton mass.
} 
mass estimates obtaining $M\left(<R_{\max }=0.75 h_{70}^{-1} \mathrm{Mpc}\right)=$ $9.5_{-1.5}^{+1.8} \times 10^{14} h_{70}^{-1} M_{\odot}$ and, using a slightly different approach, $M\left(<R_{\text {vir }}=3.85 h_{70}^{-1} \mathrm{Mpc}\right)=4.5_{-1.3}^{+1.4} \times 10^{15} h_{70}^{-1} M_{\odot}$ for the region well sampled by data and for the virialized region, respectively. This makes A697 one of the most massive clusters (e.g., Girardi \& Mezzetti 2001), in agreement with the results of Dahle et al. (2002).

To compare our result with the estimate obtained via gravitational lensing we obtain a projected mass assuming that the cluster is described by a King-like mass distribution (see Sect. 3.2) or, alternatively, a NFW profile where the mass-dependent concentration parameter is taken from Navarro et al. (1997) and rescaled by the factor $1+z$ (Bullock et al. 2001; Dolag et al. 2004). We obtain $M_{\text {proj }}\left(<R=150^{\prime \prime}\right)=(1.6-1.9) \times 10^{15} h_{70}^{-1} M_{\odot}$ in agreement with that found by gravitational lensing (Dahle et al. 2002, after having considered the different cosmological model). Using the same mass distributions we compute $M\left(<R=1 h_{70}^{-1} \mathrm{Mpc}\right)=(1.2-1.3) \times 10^{15} h_{70}^{-1} M_{\odot}$ and, using $r$-band luminosity by Popesso et al. (2004), estimate $M / L=(300-330) h_{70} M_{\odot} / L_{\odot}$. This value is comparable to those reported by Carlberg et al. (1996) for clusters of similar redshifts contradicting some evidence of an unusual high mass-to-light ratio (Dahle et al. 2002).

\subsection{Internal structure}

The velocity dispersion profile rises in the central cluster region out to $\sim 0.5 h_{70}^{-1} \mathrm{Mpc}$ (see Fig. 5). This explains why, when analyzing $7(9)$ galaxies lying within $\sim 1^{\prime}\left(\sim 0.25 h_{70}^{-1} \mathrm{Mpc}\right)$, M00 found somewhat smaller values of $\sigma_{v}\left[=553(941) \mathrm{km} \mathrm{s}^{-1}\right]$ with respect to our estimate. Increasing profiles are sometimes detected in clusters (e.g., den Hartog \& Katgert 1996; Girardi et al. 1998) and might be the signature of a relaxed cluster. The presence of circular velocities in a cluster undergoing twobody relaxation in the central region leads to a rise in the velocity dispersion profile (e.g., Merritt 1987; Merritt 1988; Sharples et al. 1988; Girardi et al. 1998). The same behaviour of the profile might be due to the high efficiency of galaxy merging in the dense, central cluster region (Menci \& Fusco Femiano 1996). Alternatively, increasing velocity dispersion profiles can be explained by the presence of subclumps at different mean velocity (partially) LOS aligned in the central cluster region. As for A697, the latter hypothesis is supported by the mean velocity profile which shows a somewhat low value of $\langle v\rangle$ at about $0.5 h_{70}^{-1} \mathrm{Mpc}$ (see Fig. 5, upper panel). Instead, as a possible explanation, we exclude the presence of still remaining field galaxies incorrectly assigned to the cluster sample since the analysis of the red-galaxy population, likely less contaminated by interlopers, leads to similar $\sigma_{v}$ and $\langle v\rangle$ profiles (see Fig. 5).

The presence of velocity and spatial segregation of galaxies with respect to their colours, luminosities and morphologies is often taken as evidence of advanced dynamical evolution of the parent cluster. In fact, these segregations can be explained by the accretion of subsequent generations of infalling field galaxies and/or secondary relaxation phenomena such as dynamical friction (e.g., Biviano et al. 1992; Ellingson et al. 2001; Biviano et al. 2002; Poggianti 2004; Goto 2005). We find marginal evidence for the galaxy segregation as expected in a very evolved cluster. Rather, the difference we detect in the mean velocity between the red and the blue populations suggests the presence of a high-velocity group, mainly populated by late-type galaxies.

Concerning the presence of subgroups, we find evidence for the non-Gaussianity of the velocity distribution, but not for correlations between galaxy positions and velocities, i.e. no significant velocity gradients and Dressler-Schectman substructure. This suggests that the possible subclumps are mainly LOS aligned (see also Pinkney et al. 1996). This LOS substructure is the likely cause of the central rise of the velocity dispersion profile.

Unfortunately, our attempt to extract subgroups through the KMM procedure does not lead to robust results. Although both the velocity distributions of the samples A and B have similar significant weighted gaps, the KMM analysis gives significant results only in the case of the sample B. Moreover, for this sample we find few alternative, significant partitions (i.e., 2/3/4/5 group partitions, the 4 -mode being the most significant one). In the case of A697, we interpret the variety of these results to imply that the presence of LOS substructure is real, but we look with caution at the quantitative results of the KMM analysis without the confirmation from the correlation between positions and velocities. We find a marginal correlation only in the case of the KMM4g3 group (at $\langle v\rangle=85894 \mathrm{~km} \mathrm{~s}^{-1}$, see Sect. 3.4). This group has a very small velocity dispersion, $\sigma_{v}<100 \mathrm{~km} \mathrm{~s}^{-1}$, and a sparse galaxy distribution thus resembling a loose group (e.g., Geller \& Huchra 1983; Ramella et al. 1989; Giuricin et al. 2000) likely still forming (e.g., Giuricin et al. 1988; Diaferio et al. 1993), rather than of a relaxed core of a secondary cluster, in agreement with its large fraction of blue galaxy population. All the 2/3/4/5 group partitions detect the presence of a low velocity group, $\langle v\rangle \sim 85500 \mathrm{~km} \mathrm{~s}^{-1}$, with a velocity dispersion of $\sigma_{v}=100-200 \mathrm{~km} \mathrm{~s}^{-1}$ and all the $3 / 4 / 5$ group partitions detect the presence of a high velocity group, $\langle v\rangle \sim 87500 \mathrm{~km} \mathrm{~s}^{-1}$, with $\sigma_{v}=200-400 \mathrm{~km} \mathrm{~s}^{-1}$. The size of the main group depends on the mode of the partition $\left(\sigma_{v}=300-1100 \mathrm{~km} \mathrm{~s}^{-1}\right)$, being about the typical cluster-mass in the most significant four-group partition $\left(\sigma_{v} \sim 600 \mathrm{~km} \mathrm{~s}^{-1}\right)$.

Analysis of Chandra data gives us other indications that A697 is far from being fully relaxed. According to the $\mathrm{X}$-ray emission, the shape of the cluster appears to be elliptical $(\epsilon=0.26 \pm 0.02)$, elongated in the SSE-NNW direction $(\mathrm{PA}=164.4 \pm 2.3$ degrees $)$. The value of ellipticity is moderate, comparable to the median values recovered in wide cluster samples (e.g., Mohr et al. 1995; De Filippis et al. 2005). The direction of the elongation agrees with previous results from ROSAT and Chandra (M00; De Filippis et al. 2005; see also ROSAT HRI image by Ota \& Mitsuda 2004) and agrees with that of the inner $\mathrm{CD}$ isophotes ( $\mathrm{PA}=163$ degrees; M00) and the mass distribution recovered from gravitational lensing (see upper right plot of Fig. 24 of Dahle et al. 2002). We find that the isodensity contour map of the galaxy distribution shows an elongation toward NW in the central cluster region, too (see Sect. 4). Interestingly, the radio contour map also shows a NW elongation in both WENSS and NVSS radio images (Kempner \& Sarazin 2001, see also Fig. 2).

The new, interesting result of our X-ray study is the presence of three structures detected via wavelet analysis. The main one almost coincides with the $\mathrm{cD}$ galaxy and thus corresponds to the cluster core. The other two - one to the NW and the other to the south - are likely to be related to subgroups located in (or projected onto) the very central cluster region, roughly within $0.2 h_{70}^{-1} \mathrm{Mpc}$ from the cluster center. The NW X-ray substructure coincides with an unusual, low surface brightness extended feature, the colour of which is consistent with that of an old stellar population at this redshift (M00, see also our Fig. 1). The 2D analysis of galaxy distribution using the photometric catalog also indicates the presence of small structures in the central cluster region, but on a somewhat larger scale, roughly 
within $0.4 h_{70}^{-1} \mathrm{Mpc}$ from the cluster center. X-ray structures are not perfectly aligned along the direction of the main axis of elliptical X-ray emission, rather they partially recover (on a smaller scale) the S-shape of the 2D galaxy distribution.

\subsection{Merging scenario}

On the basis of the asymmetric cD profile, extended features, and the highly elliptical potential implied by the arc model, M00 suggested that or A697 has recently undergone a significant merger event or that its $\mathrm{CD}$ is undergoing the process of forming its extended halo.

The present study supercedes the results of M00 finding evidence for substructure in the velocity distribution, in the X-ray emission and 2D galaxy distribution. Moreover, although we confirm previous literature findings about the moderate value of ellipticity in X-ray emission, so many cluster components show an elongation toward the NW direction that the cluster asphericity is likely to be related to an important, large-scale physical phenomenon such as a cluster merger. In particular, the evidence for subclumps in the velocity distribution, the cluster elongation and the variety of substructures we detect lead us to conclude that A697 is undergoing a multiple merger event occurring mainly along the LOS, with a transverse component in the SSE-NNW direction.

The importance of the merging is still an open question. We do not detect large size substructures, we do not find any largescale correlation between positions and velocities of member galaxies and we are not able to unequivocally detect subclumps with the KMM procedure. Therefore, we might be looking at very small clumps (e.g., $0.2 h_{70}^{-1} \mathrm{Mpc}$, the typical size of smallscale substructures, Girardi et al. 1997) accreted by a very massive cluster or, alternatively, at the remnants of a very old merger (e.g., the elongation of the X-ray emission is a long-lived phenomenon, up to $5 \mathrm{Gyr}$ after the core passage; see Roettiger et al. 1996). In both cases, one would expect a good agreement between X-ray temperature and velocity dispersion, as we observe.

Alternatively, the merging might be more important/recent, but the analysis is complicated because the merging axis is very close to the LOS. This would explain the absence of a correlation between galaxy positions and velocities, while the smallsize X-ray substructures might be identified with the cores of merging systems. The absence of a cooling core supports the hypothesis that the cluster has undergone important merging phenomena (Bauer et al. 2005).

Considering the most significant result of the KMM analysis (KMM4g), the merger would concern - or would have concerned - a typical size cluster, $\sigma_{v} \sim 600 \mathrm{~km} \mathrm{~s}^{-1}$, with two smaller systems, $\sigma_{v} \sim 200-400 \mathrm{~km} \mathrm{~s}^{-1}$, that may be detected as the two X-ray substructures. The low- $\sigma_{v}$, loose group $\mathrm{KMM} 4 \mathrm{~g} 3$ would be still infalling onto the cluster since only very dense galaxy structures are destined to survive a merger event (González-Casado et al. 1994). However, a multiple merger of systems with small, roughly comparable $\sigma_{v}$ would lead to a much less massive cluster since the cluster mass contained within $R_{\text {vir }}$ goes as $\sigma_{v}^{3}$ and that contained within a fixed radius in Mpc goes as $\sigma_{v}^{2}$. This would be incompatible with the reasonable value of $M / L$ and $\beta_{\text {spec }}$ we find (but see Schindler $\&$ Müller 1993 for the enhancement of $T_{\mathrm{X}}$ during a cluster merger). Moreover, in the case of A2744, the KMM analysis is far from giving robust results. In fact, the two-group partition, which is also very significant, suggests a quite different scenario where a very massive cluster, $\sigma_{v} \sim 1100 \mathrm{~km} \mathrm{~s}^{-1}$, accretes a much smaller system, $\sigma_{v} \sim 100 \mathrm{~km} \mathrm{~s}^{-1}$.
To obtain further insights into the importance and phase of the merging event, as well as to compute a more reliable mass estimate, one would need to have more data and to sample the cluster at much larger radii. Our sampled field is not satisfactory for such an unusual, very massive cluster $\left(R_{\text {out }}=0.34 \times R_{\mathrm{vir}}\right)$. Indeed, the situation of A697 might be comparable to that of the nearby cluster A2670 ( $z \sim 0.077)$ which is similar to A697 in many features, e.g. the rise of the velocity dispersion profile in the central region (Sharples et al. 1988) and the absence of obvious X-ray substructure (as shown by an Einstein image, but see recent Chandra results by Fujita et al. 2006). Although most of A2670 properties suggested that it is a rich, relaxed cluster, subsequent analyses showed that it may consists of subclusters that are merging along the LOS. However, this evidence only appeared when sampling the full virial region of the cluster containing two hundred galaxies (Bird 1994; Hobbs \& Willmore 1997).

Finally, it is possible that the $\mathrm{cD}$ is forming with the accretion of new mass when new groups collide with the cluster (as shown by cosmological numerical simulations, G. Murante private comm.). For instance, the NW optical extended feature corresponds to a well defined X-ray structure along the direction of the present cluster elongation suggesting a connection with the present merger. The (likely) secondary nucleus in the NE direction might be the result of an older merger. This old merger might have formed the present main cluster and in all our $\mathrm{KMM}$ results both $\mathrm{cD}$ nuclei are located in the same structure.

\section{Summary and conclusions}

We present the results of dynamical analysis of the rich, X-ray luminous and hot cluster of galaxies A697, likely containing diffuse radio emission.

Our analysis is based on new redshift data for 93 galaxies, measured from spectra obtained at the TNG, in a cluster region within a radius of $\sim 1.3 h_{70}^{-1} \mathrm{Mpc}$ from the cluster center. We also use new photometric data obtained at the INT telescope in a $30^{\prime} \times 30^{\prime}$ field.

We find that A697 appears as a peak in the redshift space at $\langle z\rangle=0.282$, which includes 68 galaxies recognized as cluster members. We compute the line-of-sight (LOS) velocity dispersion of galaxies, $\sigma_{v}=1334_{-95}^{+114} \mathrm{~km} \mathrm{~s}^{-1}$, in agreement with the high average X-ray temperature $T_{X}=(10.2 \pm 0.8) \mathrm{keV}$ recovered from our analysis of Chandra data, as expected in the case of energy-density equipartition between galaxies and gas. Assuming that the cluster is in dynamical equilibrium and mass follows the galaxy distribution, the virial theorem leads to $M\left(<R_{\max }=0.75 h_{70}^{-1} \mathrm{Mpc}\right)=9.5_{-1.5}^{+1.8} \times 10^{14} h_{70}^{-1} M_{\odot}$ and $M\left(<R_{\text {vir }}=3.85 h_{70}^{-1} \mathrm{Mpc}\right)=4.5_{-1.3}^{+1.4} \times 10^{15} h_{70}^{-1} M_{\odot}$ for the region well sampled by spectroscopic data and for the virial region, respectively.

Further findings show evidence of non-complete dynamical relaxation:

- the non-Gaussianity of the velocity distribution according to different tests;

- the presence of small-size substructures in the central cluster region as shown by the X-ray emission and 2D galaxy distribution;

- the elongation of $\mathrm{X}$-ray emission ( $\mathrm{PA}=164.4 \pm 2.3$ degrees $)$ in agreement with the above $2 \mathrm{D}$ distribution and other cluster components as shown in the literature (total mass, cD envelope, radio image); 
We conclude that A697 is undergoing a complex merger event occurring mainly along the LOS, with a transverse component in the SSE-NNW direction.

That the KMM procedure gives no robust results, as well as that we detect only small size substructures and do not find any large-scale correlation between positions and velocities of member galaxies suggest that we might be looking at very small clumps accreted by a very massive cluster or at the remnants of a very old merger. The merging process might be more important/recent, but the analysis is complicated because the merging axis is very close to the LOS. To obtain further insights into the importance and phase of the merging event one would need to have more data and sample the cluster at much larger radii.

The spatial correlation between the (likely) radio halo and the X-ray and optical cluster structure supports the hypothesis of a connection between extended radio emission and merging phenomena.

Acknowledgements. We thank Joshua A. Kempner for helpful suggestions and Andrea Biviano, Giuseppe Murante, and Mario Nonino for interesting discussions. We thank the referee, Hans Böhringer, for his useful suggestions.

This publication is based on observations made on the island of La Palma with the Italian Telescopio Nazionale Galileo (TNG), operated by the Fundación Galileo Galilei - INAF (Istituto Nazionale di Astrofisica), and with the Isaac Newton Telescope (INT), operated by the Isaac Newton Group (ING), in the Spanish Observatorio of the Roque de Los Muchachos of the Instituto de Astrofisica de Canarias.

This publication also makes use of data obtained from the Chandra data archive at the NASA Chandra X-ray center (http: //asc. harvard.edu/cda/).

This work was partially supported by a grant from the Istituto Nazionale di Astrofisica (INAF, grant PRIN-INAF2005 CRA ref number 1.06.08.05).

\section{References}

Abell, G. O., Corwin, H. G. Jr., \& Olowin, R. P. 1989, ApJS, 70, 1

Ashman, K. M., Bird, C. M., \& Zepf, S. E. 1994, AJ, 108, 2348

Barrena, R., Biviano, A., Ramella, M., Falco, E. E., \& Seitz, S. 2002, A\&A, 386, 816

Bauer, F. E., Fabian, A. C., Sanders, J. S., Allen, S. W., \& Johnstone, R. M. 2005, MNRAS, 359, 1481

Beers, T. C., Flynn, K., \& Gebhardt, K. 1990, AJ, 100, 32

Beers, T. C., Forman, W., Huchra, J. P., Jones, C., \& Gebhardt, K. 1991, AJ, 102, 1581

Bertin, E., \& Arnouts, S. 1996, A\&AS, 117, 393

Bird, C. M. 1994, ApJ, 422, 480

Bird, C. M., \& Beers, T. C. 1993, AJ, 105, 1596

Biviano, A., Girardi, M., Giuricin, G., Mardirossian, F., \& Mezzetti, M. 1992, ApJ, 396, 35

Biviano, A., Katgert, P., Thomas, T., \& Adami, C. 2002, A\&A, 387, 8

Böhringer, H., \& Schuecker, P. 2002, in Merging Processes in Galaxy Clusters, ed. L. Feretti, I. M. Gioia, \& G. Giovannini (The Netherlands: Kluwer Ac. Pub.), Observational signatures and statistics of galaxy cluster mergers, in press

Bonamente, M., Joy, M. K., LaRoque, J., et al. 2005, ApJ, submitted [arXiv: astro-ph/0512349]

Boschin, W., Girardi, M., Barrena, R., et al. 2004, A\&A, 416, 839

Boschin, W., Girardi, M., Spolaor, M., \& Barrena, R. 2006, A\&A, 449, 461

Bullock, J. S., Kolatt, T. S., Sigad, Y., et al. 2001, MNRAS, 321, 559

Buote, D. A. 2002, in Merging Processes in Galaxy Clusters, ed. L. Feretti, I. M. Gioia, \& G. Giovannini (The Netherlands: Kluwer Ac. Pub.), Optical Analysis of Cluster Mergers, in press

Burgett, W. S., Vick, M. M., Davis, D. S., et al. 2004, MNRAS, 352, 605

Burns, J. O., Roettiger, K., Ledlow, M., \& Klypin, A. 1994, ApJ, 427, L87

Burstein, D., \& Heiles, C. 1982, AJ, 87, 1165

Buta, R., \& Williams, K. L. 1995, AJ, 109, 543

Buta, R., Mitra, S., de Vaucouleurs, G., \& Corwin, H. G., Jr. 1994, AJ, 107, 118

Carlberg, R. G., Yee, H. K. C., \& Ellingson, E., et al. 1996, ApJ, 462, 32

Carlberg, R. G., Yee, H. K. C., \& Ellingson, E. 1997, ApJ, 478, 462

Colless, M., \& Dunn, A. M. 1996, ApJ, 458, 435

Condon, J. J., Cotton, W. D., Greisen, E. W., et al. 1998, AJ, 115, 1693
Cousins, A. W. J. 1976, MemRAS, 81, 25

Crawford, C. S., Edge, A. C., Fabian, A. C., et al. 1995, MNRAS, 274, 75

Dahle, H., Kaiser, N. Irgens, R. J., Lilje, P. B., \& Maddox, S. J. 2002, ApJS, 139, 313

Danese, L., De Zotti, C., \& di Tullio, G. 1980, A\&A, 82, 322

De Filippis, E., Sereno, M., Bautz, M. W., \& Longo, G. 2005, ApJ, 625, 108

den Hartog, R., \& Katgert, P. 1996, MNRAS, 279, 349

Diaferio, A., Ramella, M., Geller, M. J., \& Ferrari, A. 1993, AJ, 105, 2035

Dickey, J. M., \& Lockman, F. J. 1990, ARA\&A, 28, 215

Dolag, K., Bartelmann, M., Perrotta, F., et al. 2004, A\&A, 416, 853

Dressler, A., \& Shectman, S. A. 1988, AJ, 95, 985

Ebeling, H., Edge, A. C., Böhringer, H., et al. 1998, MNRAS, 301, 881

Ellingson, E., Lin, H., Yee, H. K. C., \& Carlberg, R. G. 2001, ApJ, 547, 609

Fadda, D., Girardi, M., Giuricin, G., Mardirossian, F., \& Mezzetti, M. 1996, ApJ, 473,670

Fasano, G., \& Franceschini, A. 1987, MNRAS, 225, 155

Feretti, L. 1999, MPE Report No. 271

Feretti, L. 2002, The Universe at Low Radio Frequencies, Proceedings of IAU Symposium 199, held 30 Nov.-4 Dec. 1999, Pune, India, ed. A. Pramesh Rao, G. Swarup, \& Gopal-Krishna, 133

Feretti, L. 2005, X-Ray and Radio Connections, ed. L. O. Sjouwerman, \& K. K. Dyer, Published electronically by NRAO, http://www.aoc.nrao.edu/ events/xraydio, Held 3-6 February 2004 in Santa Fe, New Mexico, USA

Feretti, L., Gioia, I. M., \& Giovannini, G. 2002, Merging Processes in Galaxy Clusters (The Netherlands: Kluwer Ac. Pub.)

Ferrari, C., Benoist, C., Maurogordato, S., Cappi, A., \& Slezak, E. 2005, A\&A, 430, 19

Fujita, Y., Sarazin, C. L., Reiprich, T. H., et al. 2004, ApJ, 616, 157

Fujita, Y., Sarazin, C. L., Sivakoff, G. R., \& Gregory, R. 2006, PASJ, 58, 131

Gebhardt, K., \& Beers, T. C. 1991, ApJ, 383, 72

Geller, M. J., \& Beers, T. C. 1982, PASP, 94, 421

Geller, M. J., \& Huchra, J. P. 1983, ApJS, 52, 61

Giovannini, G., \& Feretti, L. 2002, in Merging Processes in Galaxy Clusters, ed. L. Feretti, I. M. Gioia, \& G. Giovannini (The Netherlands: Kluwer Ac. Pub.), Diffuse Radio Sources and Cluster Mergers

Giovannini, G., Feretti, L., Venturi T., Kim, K.-T., \& Kronberg, P. P. 1993, ApJ, 406, 399

Giovannini, G., Tordi, M., \& Feretti, L. 1999, New Astron., 4, 141

Girardi, M., \& Mezzetti, M. 2001, ApJ, 548, 79

Girardi, M., \& Biviano, A. 2002, in Merging Processes in Galaxy Clusters, ed. L. Feretti, I. M. Gioia, \& G. Giovannini (The Netherlands: Kluwer Ac. Pub.), Optical Analysis of Cluster Mergers

Girardi, M., Biviano, A., Giuricin, G., Mardirossian, F., \& Mezzetti, M. 1993, ApJ, 404, 38

Girardi, M., Fadda, D., Giuricin, G., et al. 1996, ApJ, 457, 61

Girardi, M., Escalera, E., Fadda, D., et al. 1997, ApJ, 482, 41

Girardi, M., Giuricin, G., Mardirossian, F., Mezzetti, M., \& Boschin, W. 1998, ApJ, 505, 74

Giuricin, G., Marinoni, C., Ceriani, L., \& Pisani, A. 2000, ApJ, 543, 178

Giuricin, G., Gondolo, P., Mardirossian, F., Mezzetti, M., \& Ramella, M. 1988, A\&A, 199, 85

Gómez, P. L., Hughes, J. P., \& Birkinshaw, M. 2000, ApJ, 540, 726

González-Casado, G., Mamon, G., \& Salvador-Solé, E. 1994, ApJ, 433, L61

Govoni, F., Ensslin, T. A., Feretti, L., \& Giovannini, G. 2001a, A\&A, 369, 441

Govoni, F., Feretti, L., Giovannini, G., et al. 2001b, A\&A, 376, 803

Goto, T. 2005, MNRAS, 359, 1415

Gullixson, C. A. 1992, in Astronomical CCD Observing and Reduction Techniques, ed. S. B. Howell, ASP Conf. Ser., 23, 130

Hanisch, R. J. 1982, A\&A, 116, 137

Henry, J. P., Finoguenov, A., \& Briel, U. G. 2004, ApJ, 615, 181

Hobbs, I. S., \& Willmore, A. P. 1997, MNRAS, 289, 685

Jones, C., \& Forman, W. 1999, ApJ, 511, 65

Johnson, H. L., \& Morgan, W. W. 1953, ApJ, 117, 313

Kempner, J. C., \& David, L. P. 2004, MNRAS, 349, 385

Kempner, J. C., \& Sarazin, C. L. 2001, ApJ, 548, 639

Kennicutt, R. C. 1992, ApJS, 79, 225

Kriessler, J. R., \& Beers, T. C. 1997, AJ, 113, 80

Ledermann, W. 1982, Handbook of Applicable Mathematics (New York: Wiley), Vol. 6

Limber, D. N., \& Mathews, W. G. 1960, ApJ, 132, 286

López-Cruz, O., Barkhouse, W. A., \& Yee, H. K. C. 2004, ApJ, 614, 679

Markevitch, A., Gonzalez, A. H., David, L., et al. 2002, ApJ, 567, 27

Markevitch, M., \& Vikhlinin, A. 2001, ApJ, 563, 95

Mazure, A., Katgert, P., den Hartog, P., et al. 1996, A\&A, 310, 31

Menci, N., \& Fusco-Femiano, R. 1996, ApJ, 472, 46

Mercurio, A., Girardi, M., Boschin, W., Merluzzi, P., \& Busarello, G. 2003, A\&A, 397, 431

Merritt, D. 1987, ApJ, 313, 121 
Merritt, D. 1988, in The Minnesota Lectures on Clusters of Galaxies and LargeScale Structures, ed. J. M. Dickey, ASP Conf. Ser., 5, 175

Metzger, M. R., \& Ma, C.-P. 2000, AJ, 120, 2879 (M00)

Mohr, J. J., Evrard, A. E., Fabricant, D. G., \& Geller, M. J. 1995, ApJ, 447, 8

Mohr, J. J., Geller, M. J., Fabricant, D. G., et al. 1996, ApJ, 470, 724

Navarro, J. F., Frenk, C. S., \& White, S. D. M. 1997, ApJ, 490, 493

Ota, N., \& Mitsuda, K. 2004, A\&A, 428, 758

Pinkney, J., Roettiger, K., Burns, J. O., \& Bird, C. M. 1996, ApJS, 104, 1

Pisani, A. 1993, MNRAS, 265, 706

Poggianti, B. M. 1997, A\&AS, 122, 399

Poggianti, B. M. 2004, Proceedings of Baryons in Dark Matter Halos, Novigrad,

Croatia, 5-9 Oct. 2004, ed. R. Dettmar, U. Klein, \& P. Salucci, Published by SISSA, Proceedings of Science, http://pos.sissa.it, p.104.1

Popesso, P., Böhringer, H., Brinkmann, J., Voges, W., \& York, D. G. 2004, A\&A, 423, 449

Press, W. H., Teukolsky, S. A., Vetterling, W. T., \& Flannery, B. P. 1992, in Numerical Recipes (Second Edition) (Cambridge University Press)

Raymond, J. C., \& Smith, B. W. 1977, ApJS, 35, 419

Ramella, M., Geller, M. J., \& Huchra, J. P. 1989, ApJ, 344, 57
Roettiger, K., Burns, J. O., \& Loken C. 1996, ApJ, 473, 651

Roettiger, K., Loken, C., \& Burns, J. O. 1997, ApJS, 109, 307

Röttgering, H., Snellen, I., Miley, et al. 1994, ApJ, 436, 654

Sarazin, C. L. 2002, in Merging Processes in Galaxy Clusters, ed. L. Feretti, I. M. Gioia, \& G. Giovannini (The Netherlands: Kluwer Ac. Pub.), The Physics of Cluster Mergers

Schindler, C. L., \& Müller, E. 1993, A\&A, 272, 137

Schuecker, P., Böhringer, H., Reiprich, T. H., \& Feretti, L. 2001, A\&A, 378, 408

Shapiro, S. S., \& Wilk, M. B. 1965, Biometrika, 52, 591

Sharples, R. M., Ellis, R. S., \& Gray, P. M. 1988, MNRAS, 231, 479

Slezak, E., Durret, F., \& Gerbal, D. 1994, AJ, 108, 1996

Sun, M., Murray, S. S., Markevitch, M., \& Vikhlinin, A. 2002, ApJ, 565, 867

The, L. S., \& White, S. D. M. 1986, AJ, 92, 1248

Tonry, J., \& Davis, M. 1979, AJ, 84, 1511

Tribble, P. 1993, MNRAS, 263, 31

Vikhlinin, A., Forman, W., \& Jones, C. 1997, ApJ, 474, 7

Wainer, H., \& Schacht, S. 1978, Psychometrika, 43, 203

White, D. A. 2000, MNRAS, 312, 663 